\title{
The Advances of Neutrophil-Derived Effective Drug Delivery Systems: A Key Review of Managing Tumors and Inflammation
}

\author{
Huaiji Wang' \\ Jie Zang ${ }^{2}$ \\ Zihan Zhao ${ }^{3}$ \\ Qin Zhang $\mathbb{D}^{\prime}$ \\ Shunjie Chen'
}

'Department of Nephrology, Shanghai Fourth People's Hospital, School of Medicine, Tongji University, Shanghai, People's Republic of China; ${ }^{2}$ The Institute for Biomedical Engineering \& Nano Science, School of Medicine, Tongji University, Shanghai, People's Republic of China; ${ }^{3}$ Department of Dermatology, Shanghai Tenth People's Hospital, School of Medicine, Tongji University, Shanghai, People's Republic of China
Correspondence: Shunjie Chen Department of Nephrology, Shanghai Fourth People's Hospital, School of Medicine, Tongji University, Shanghai, People's Republic of China

Email chenshunjie77csj@163.com

\begin{abstract}
The chimeric trait of recruitment by inflammatory signals endows neutrophils with the functionality of migrating to inflamed tissues, which can be utilized to tailor novel drug delivery systems. In this review, we introduce a mechanism of neutrophil-derived drug delivery systems recruited into inflamed sites and provide insight into tumors and inflammation therapy. In particular, the advantages of neutrophils - their endogenous-derived neutrophil membrane, exosomes as drug carriers for augmented targeting, prolonged circulation, and improved biostability - were concluded. Subsequently, the latest application in the treatment of tumors and inflammation was elaborated upon, followed by a discussion of the future prospects to neutrophil-derived delivery systems. This promising system will provide new therapeutic avenues for the treatment of inflammation and tumors.

Keywords: neutrophil-derived delivery, tumors and inflammation, targeting, circulation, biostability
\end{abstract}

\section{Introduction}

Inflammation response plays a significant role in the innate and adaptive response in the body against infection and tissue damage, characterized by vascular response, the recruitment of immune cells, and the release of molecular mediators. ${ }^{1,2}$ When subjected to detrimental stimuli, the immune system will be quickly activated to eliminate pathogens, remove the necrotic cells and damaged tissues, followed by the initiation of tissue repair. ${ }^{3}$ If the inflammatory stimulus exists persistently or compromises the inflammatory regulation mechanism, the occurrence of a series of diseases related to inflammation will be inevitable, such as arthritis, atherosclerosis, and tumors. ${ }^{4}$ During the development of inflammation or tumors, cytokines secreted from the inflamed tissues will recruit substantial cells that induce the formation of a tumor inflammatory microenvironment. Meanwhile, neutrophils - a subset of immune cells - follow chemokine signals to cross the vascular endothelium and migrate to the site of inflammation or tumor. ${ }^{5,6}$

The complex microenvironment in vivo possesses the challenge for drugs to arrive at the sites of inflammation and tumors effectively. ${ }^{7,8}$ As a result, it has been a persistent dilemma pondered by researchers on how to accurately deliver drugs to the sites of inflammation or tumors to minimize the toxic effects and maximize the therapeutic one. ${ }^{9,10}$ Currently, therapeutic drugs used in clinics display shortcomings such as poor solubility and stability, leading to rapid elimination after 
intravenous injection, as well as the irregular biodistribution contributing to toxicity in normal tissues. ${ }^{11}$ In this context, nanodrugs, as drug delivery systems constructed to operate at the nanometer size range with novel engineered properties - such as improved drug solubility, prolonged blood circulation, increased biocompatibility, and decreased toxicity - have been approved for the treatment of infections and cancer in clinics. ${ }^{12-15}$ Despite these remarkable achievements, undesired targeting is envisaged as a major challenge hampering accumulation lying in the drug delivery system. ${ }^{16-18}$ Researchers have attempted to prolong circulation and enhance targeting of nanodrugs by modifying negatively charged materials on the surface of nanoparticles to attenuate non-specific binding to cells in blood vessels. ${ }^{19,20}$ Under the condition of promoting circulation, polyethylene glycol (PEG) was also exploited and polypeptides or antibodies were used for specifically binding to receptors overexpressed in tumor tissues or inflammatory sites for amplifying active targeting. ${ }^{21-24}$ However, the foregoing strategies are subject to the limits of complex material synthesis and carrier design.

With the development of nanobiotechnology in recent years, delivery systems based on endogenous cells have gradually attracted wide attention, especially for living cell delivery systems such as neutrophils. ${ }^{25-28}$ Neutrophilbased delivery systems can imitate the natural mechanism of the immune system involved in inflammation to circumvent these limitations of traditional nanoparticles in low biocompatibility, short circulation and poor immunogenicity-opening a new avenue to inflammation and tumor targeting. ${ }^{7,29,30}$ Attributed to toxicity of chemotherapeutic drugs to cell carrier, directly using immune cells as drug delivery vehicles poses certain challenges. Thus, it is an urgent need to tailor safer and more effective drug delivery systems. The combination of living cells and nanodrugs is capable of effectively avoiding the obstacle, showing broad application prospects in the nanotheranostics of diseases. ${ }^{31-34}$ In addition to living cells, the biomimetic cell membrane strategy has also attracted substantial attention. Isolated from living cells, cell membranes inherited the functionality of source cells and were coated onto drug-loading nanoparticles for the purpose of reducing clearance by immune cells and prolonged circulation. Due to the utterly retained cell membrane componentsthe integrated adhesion molecule system - the obtained biomimetic nanoparticles can exert the function of the intercellular communication. Neutrophil membrane biomimetic nanoparticles have realized targeting capacity by adhering to activated endothelium via the interaction between integrin $\beta 2$ on neutrophils and ICAM-1 expressed on endothelium. In this context, some researchers have successfully developed neutrophil-membrane-coated nanodrug delivery systems, which inherit the hallmark of neutrophils in inflammation targeting and tissue penetration. $^{35-37}$ This biomimetic strategy is widely used because of the brief preparation process, that is, the prepared cell membrane is coated on the surface of the nanomaterial by extrusion. ${ }^{38}$ Another biological carrier derived from neutrophils are exosomes-like the neutrophil membrane delivery system - that also have the comparable hallmarks of low immunogenicity, prolonged circulation, and deep penetration. ${ }^{39}$ In recent studies, much important information involved in neutrophil exosomes has been unveiled. Exosomes inherit multiple bioactive molecules from their mother cells. For example, Exos isolated from T-cell-derived cells containing T-cell receptors, CAR-T cells secreted Exos, are enriched with CAR and cancer cell Exos expressing PD-L1. It has been reported that $\mathrm{N}$-formylmethionyl-leucyl-phenylalanine (fMLP)-induced neutrophil exosomes display inhibitory effects on dendritic cells - contributing to an attenuated capacity for T-cell proliferation. Chemotactic activity of mother neutrophils can be elicited by neutrophil exosomes in inflammation, which carry leukotriene B4 upon N-fMLP stimulation. As a result, neutrophil exosomes can deliver signals to recipient cells, potentially exerting significant effects on the management of inflammation and cancer.

Cell-based drug delivery systems have attracted substantial attention in recent years. In this review, we mainly focus on neutrophil-derived effective drug delivery systems on managing tumors and inflammation. A mechanism of neutrophils recruited into inflamed tissue and tumors was introduced. After highlighting the advantages of neutrophils, neutrophil membranes, and neutrophil exosomes as drug delivery vehicles (Figure 1), the latest application progress in tumors and inflammation therapy was illustrated. Lastly, we discussed the future prospects of neutrophil-derived delivery systems, with a specific emphasis on this promising strategy.

\section{Strategies for Neutrophil-Derived Delivery Systems Neutrophil Loading Drug Delivery Systems}

Neutrophils, as important cells in the innate immune system, are the most abundant type of white blood cell 


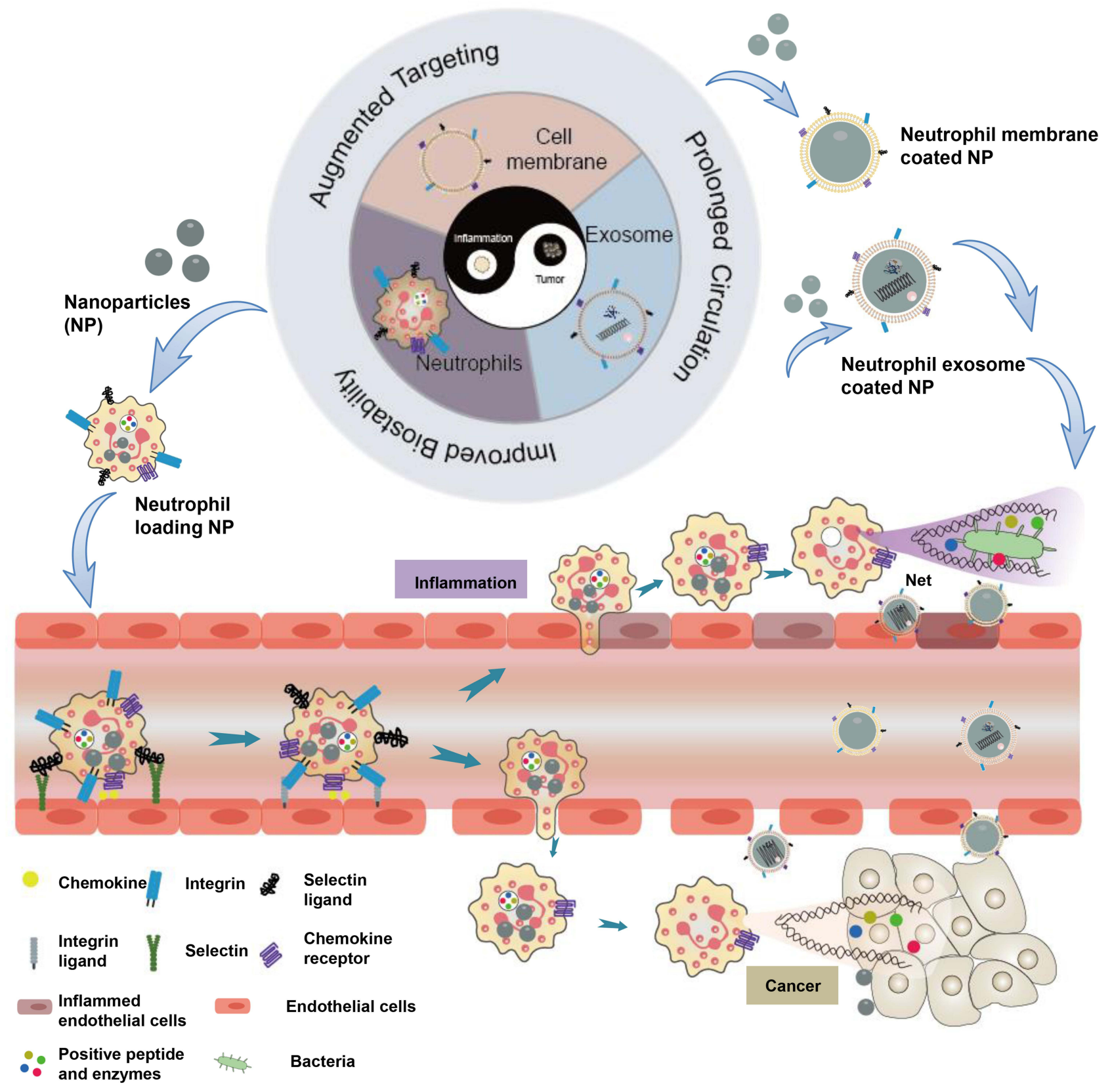

Figure I Schematic illustration of tumor and inflammation therapies by employing neutrophils, cell membrane and exosome as nanodrug delivery system, with the advantages in augmented targeting, prolonged circulation and improved biostability.

in the body. In healthy adults, around 20,000 neutrophils are produced each day by bone marrow stem cells, accounting for $50-70 \%$ of white blood cells. ${ }^{28}$ The generation of neutrophils is controlled by granulocyte colony stimulating factor (G-CSF), in response to interleukin-17A (IL-17A) synthesized by $\mathrm{T}$ cells, which regulate neutrophils. As the first responder to inflammatory signals, neutrophils are expected to pass through blood vessels along with interstitial tissues under the guidance of cytokines, and then migrate to the site of infection or inflammation within several minutes. When circulating, mature neutrophils with an average diameter of 7-10 $\mu \mathrm{m}$ display segmented nuclei, and their cytoplasm is enriched with granules as well as secretory vesicles. After releasing antibacterial substances, including reactive oxygen species (ROS) and hydrolase, neutrophils are able to eradicate invaded pathogens and reduce inflammation in the body. ${ }^{3,40}$ Neutrophils actively home to the site of tissue injury or infection, along the concentration gradient of chemokines, penetrating endothelial 
cells into non-vascular areas-named as chemotaxis. ${ }^{41}$ The principle of targeted enrichment to the sites of inflammation or tumor is shown in Figure 1: 1) under the recruitment of chemokines (such as CINC, CXCL2), to which chemokine receptors on the surface of neutrophils will bind; 2) neutrophils migrate through the binding of selectin ligand on the surface of neutrophils and selectin on endothelial cells; 3 ) integrin onto neutrophil binding to integrin ligand (CAMs) to achieve endothelial cell adhesion; 4) neutrophils migrate through the gap between endothelial cells to the site of inflammation or tumor, release net and particles for antivirus or anti-tumor. ${ }^{3} \mathrm{At}$ present, researchers have found that neutrophil infiltration has existed in a variety of tumor models. With neutrophils as the target, the design of neutrophil loading drug delivery systems is not only expected to remedy the insufficiencies of nanodrugs, but also show certain prospects in the treatment of inflammation and tumors. ${ }^{28}$ Ultimately, neutrophils display huge potential in drug delivery due to the characteristics of prolonged circulation, abundant surface receptors, flexible morphology, and no immunogenicity-which overcome the limits of traditional nanocarriers. ${ }^{42-44}$ The utilization of neutrophils as a nanodrug delivery vehicle was reported in 2017 for the treatment of glioma after surgery and is noteworthy. ${ }^{44}$ Subsequently, drug-carrying neutrophil technology was used to load nanomaterials with different functions, which were applied to the treatment of various inflammation and tumors.

\section{Neutrophil Membrane Biomimetic Drug Delivery Systems}

Instead of using living cells, neutrophil membrane camouflaging nanoparticles provide a unique platform for achieving the specific delivery of therapeutics in the management of cancer and inflammation. In the cell membrane biomimetic nanosystem, the neutrophil membranes were separated and purified to coat on the surface of the nanoparticles. ${ }^{45}$ Because of the utterly retained cell membrane components - the integrated adhesion molecule system-the obtained biomimetic nanoparticles can inherit the biological activity of the source cells and exert the function of the intercellular communication. ${ }^{46}$ Owing to origin from the endogenous cells, the neutrophil membrane camouflaging nanosystem cannot only prolong the circulation time adverse, but also improve the biocompatibility and targeting. ${ }^{38,47,48}$
Besides, adverse effects - like the toxicity to organscan also be impaired because of the extraordinary biocompatibility. In light of the above advantages, neutrophilmembrane-coated nanosystems have been universally used in the treatment of inflammation and tumors. ${ }^{49,50}$ Different from living cells with more attention to activity and storage conditions, cell membrane biomimetic nanoparticles have similar storage conditions with traditional chemically synthesized drugs like liposome. However, the current challenge is how to retain the designed delivery system with all receptors and adhesion molecules on the surface of neutrophils transferring to nanoparticles and subsequent release after arriving at the inflammatory lesions so that they have similar functions with neutrophils. The first research on neutrophil membrane coating nanodrug delivery was reported in 2017, and has greatly expanded thereafter. ${ }^{50}$

\section{Neutrophil Exosome-Inspired Drug Delivery Systems}

Recent studies have shown that neutrophil-derived exosomes can play a role independently outside neutrophils and orchestrate adaptive immune responses by influencing some cell types. Exosomes, as endogenous extracellular vesicles with nanoscales, act as messengers between cells and organs due to their rich proteins, lipids, and nucleic acids. They display similarity to the cell membrane in terms of negative charge, nontoxicity, and low immunogenicity. Therefore, exosomes have the advantages of avoiding phagocytosis, prolonging circulation, and penetrating deep tissues-showing great potential as drug delivery vehicles. Natural exosomes have a certain degree of heterogeneity; that is, the components of exosomes are closely correlated with their source cells with specific functions. Immune cells, such as exosomes secreted by neutrophils, target tumors and inflammation. ${ }^{39,51}$ The construction of neutrophil-derived exosome systems has become a bright spot in the field of drug delivery.

\section{Advantages of Neutrophil-Derived Drug Delivery Systems}

Active Targeting in Tumors and Inflammation: For drug delivery, targeting is the pivotal factor determining the therapeutic effect. As is known, a small molecule drug with a hydrophobic structure has poor solubility, and is expected to distribute irregularly, leading to toxicity toward major organs. With the development of nanotechnology, the aforementioned drawbacks have been 
improved. ${ }^{52}$ By wrapping with nanomaterials, drug solubility can be increased with desired distribution in vivo, which is beneficial for subsequent accumulation. ${ }^{53,54}$ Taking tumors, for example, nanoparticles with passive targeting can accumulate in the tumor tissue after the intravenous injection. To augment targeting efficiency, a couple of molecules can be modified onto nanoparticles, such as folate, ${ }^{55} \mathrm{RGD},{ }^{56} \mathrm{PD}-\mathrm{L} 1,{ }^{57}$ etc. For inflammation, nanoparticles are also decorated with ligands that bind to receptors around inflammatory tissues. However, there appears to be some controversy in terms of nanoparticle targeting at tumors in vivo, although plenty of research has reported excellent targeting based on animal experiments. Due to the difference between animals and humans, clinical experiments of nanodrugs targeting accumulating in tumors have failed to get breakthrough. Most studies have unveiled that nanoparticles will be adsorbed on the surface by plasma proteins and other biomolecules, forming a dynamic biomolecule corona. ${ }^{58}$ In this context, nanoparticles will be recognized and eliminated by macrophages, known as opsonization, which may lead to reduced accumulation in the targeting site. ${ }^{59}$ Fortunately, these obstacles can be bypassed by employing neutrophil-loading nanodrugs and retransforming them into the body. As endogenous immune cells, neutrophils could avoid invasion by the immune system. Beneficial from the recruitment by chemokines, neutrophil-loading nanodrugs can actively accumulate in the tumor. ${ }^{28,44}$ Neutrophil membrane and exosome-coated nanoparticles can also escape immune recognition by camouflaging and accumulate in the targeting site due to the inherited function from the mother cell-similar protein as well as lipid structure. ${ }^{39,49}$

Prolonged Circulation in Blood: The prerequisite for drugs accumulating in the desired site of disease is the capability of prolonged circulation. ${ }^{60}$ Small molecule drugs are easy to aggregate due to poor solubility and biological drugs tend to be degraded after administration-leading to a short half-life. After being encapsulated by nanoparticles, the solubility and biodistribution of drugs were improved due to their excellent biocompatibility. ${ }^{13}$ For instance, biocompatible albumin nanoparticles were selected as the candidate for loading hydrophobic drugs. ${ }^{61}$ To promote the level of circulation, PEG-which helps to increase solubility_was employed widely. However, recent research shows unsatisfactory outcomes of circulation by PEG coating. ${ }^{62}$ Fortunately, neutrophil-derived drug delivery systems provide an alternative to prolonging circulation. As endogenous cells, neutrophils carrying nanodrugs will not be eliminated by the immune system when retransforming. Besides, neutrophil membrane-coated nanoparticles inherit the function of source cells to avoid immune elimination due to CD47 molecules on the surface-a "don't eat me" signal. ${ }^{63}$ In this case, most biomimetic nanoparticles could survive, and the circulation could be prolonged. ${ }^{64,65}$ Exosomes secreted by neutrophils, another inherited functionality of neutrophils, are being widely reported for their biocompatibility, which is beneficial to circulation.

Biological Stability in Blood: The necessity of long-term storage of drugs suggests that it is vital to sustaining the biological stability of drugs. When injected, the aggregation and changed structure of drugs in the blood would be frustrated. Although the stability of these drugs - such as small molecule drugs, siRNA, or anti-PD-1 antibody-can be improved after being encapsulated with nanoparticles; the challenge in the present study lies in exploiting a general strategy to realize the goal of synthesizing stable nanodrugs due to the diversity of nanomaterials in the stability. ${ }^{66-68}$ It has been demonstrated that some nanoparticles (such as protein nanoparticles) loading chemotherapy drugs would attenuate the toxicity generated by bare drugs to neutrophils after phagocytosis. Intriguingly, nanodrugs internalized by neutrophils would experience on-demand release in the targeting site because of the stimulation by inflammation signals. ${ }^{44}$ During this period, neutrophils deliver a net that also serves to fight with inflammation cells. ${ }^{40}$ There hence, this tactic by neutrophil internalizing nanodrugs was effective in maintaining stability in the complicated milieu of blood. Distinguishing from loading neutrophils with nanoparticles and coating nanoparticles with neutrophil membranes or exosomes was a biomimetic approach that seemed to dress nanoparticles in brand-new clothes. The similar structure of the phospholipid bilayer on the surface of hybrid nanoparticles is beneficial to stability. Importantly, various types of nanoparticles can be coated in this manner, such as inorganic materials, polymers, motel nanoparticles, etc. $^{69}$

\section{Application of Neutrophil-Derived Drug Delivery Systems in Tumors and Inflammation \\ Neutrophil-Derived Drug Delivery Systems in the Treatments of Tumors Neutrophil Loading Drug Delivery System}

Two aspects could be considered to enhance the targeting efficiency entailing the targeting of neutrophils and 
nanosystems. For increasing the targeting ability of neutrophils, recent strategies mainly focus on facilitating the recruitment of chemokines by surgery, irradiation, and photothermal therapy (PTT) to induce the inflammation microenvironment. For example, based on in-situ resection to amplify the inflammation signal for recruiting neutrophils to cross the blood-brain barrier, liposomes carrying paclitaxel (PTX) were loaded into neutrophils (PTX-CL /NEs) and injected into mice for the therapy of glioma. It was found that no obvious drug release happened within 8 h. PTX-CL/NEs exhibited high fluorescence intensity compared to other groups observed by the imaging system, indicative of PTX-CL/NEs possessing the functionality of normal cells to bypass the blood-brain barrier to penetrate the tumor tissue. The delivery system could slow down the growth and recurrence of glioma and increase the survival rate. $^{44}$ Apart from surgery to prompt the inflamed signal, utilizing PTT to enlarge the inflamed signal for the recruitment of drug-loaded neutrophils was used for active targeting therapy. Slight radiation would not generate direct damage to the tumor but would increase the release of inflammatory factors responsible for inducing neutrophils to migrate to the tumor tissue (Figure 2A). In this study, PEG-modified Au nanorods were selected as photosensitive materials, and neutrophils loading PTX-carried liposomes were used for chemotherapy. After PTT, neutrophils could accumulate more in the tumor, increasing anti-tumor outcomes and reducing systemic toxicity, advantageous for clinical transformation of the in vitro reinfusion strategy of neutrophil-based drug delivery systems. The release of chemokines that induced neutrophil migration, abraxane, was loaded into neutrophils (Abraxane/NEs) for treatment in the SNU-719-bearing tumor model. Combining with local radiation, Abraxane/NEs displayed more distribution in the tumor as well as improved therapy, with no adverse effect (Figure $2 \mathrm{~B}$ and $\mathrm{C}$ ). ${ }^{70}$ In addition to the abovementioned method, creating an inflamed microenvironment to increase targeted accumulation in the tumor and remolding neutrophils with the strong chemotaxis seemed to be attractive measures. An interesting report illustrated that targeting would be more precise if neutrophils underwent immune training by changing the local living environment to strengthen immune cell activity. The results showed that neutrophils harvested from CpG-treated mice exhibited significant accumulation in the tumor. This strategy displayed a promising application due to its compelling biocompatibility and re-production. ${ }^{71}$
Another alternative to prompt the targeting efficiency of neutrophil-based systems was to endow nanoparticles with targeting. A recent report demonstrated that a neutrophil-based multistage drug delivery system was developed for lung cancer by PTT. In its design, targeting peptide RGD-modified Au nanorods were incubated with neutrophils, which strikingly enhanced the homing efficiency of tumors. Subsequently, multifunctional nanorods released by neutrophils were able to penetrate deep tissue, contributing to the suppression of tumors (Figure 2D and E). ${ }^{72}$

Although neutrophils have been exploited as drug carriers to overcome multiple physiological barriers during delivery, the crucial point is how to develop a universal approach for neutrophil phagocytizing nanodrugs. Based on a previous report that pathogen-like bacteria could be recognized and phagocytized by neutrophils, a pristine strategy of mimicking pathogens was designed for neutrophil targeting. Bacterial outer membrane vesicles were used to encapsulate nanoparticles, which were recognized and phagocytized by neutrophils due to some molecular patterns of the pathogens in the bacteria (Figure 3A). The biomimetic system could migrate to the tumor after PTT, releasing cis-platinum loaden nano-pathogenoid (NPNs@Pt) to kill residual tumor cells and realize complete removal of the tumor. Figure 3B displayed more accumulation in the tumor site after PTT compared to the control, indicating the precise targeting of neutrophils. Undergoing combined PTT twice, the tumor tissues of the mice can be completely eliminated, with no recurrence seen during observation, which was ascribed to the increase of NPNs@Pt enriching in the tumor, inducing the elimination of tumor cells (Figure 3C). This strategy can be widely applied to the specific targeting of different nano-microparticles to neutrophils and the treatment of various types of diseases, such as tumors or inflammation involving neutrophils. ${ }^{73}$

In addition to constructing a delivery system by incubating cells with nanomaterials, a novel tactic by hitchhiking was utilized to decrease negativity in the safety and viability of neutrophils due to operation in vitro. Compared with retransformation, neutrophil hitchhiking was easier to realize without worry during cell extraction and retransformation. Bovine serum albumin (BSA) nanoparticles were loaded with pyrochlorophyll a, in virtue of the Fc $\gamma$ receptor in neutrophils that recognized and phagocytosed BSA nanoparticles (BSA NPs), in which case neutrophils carried BSA NPs to the tumor site. In 
A

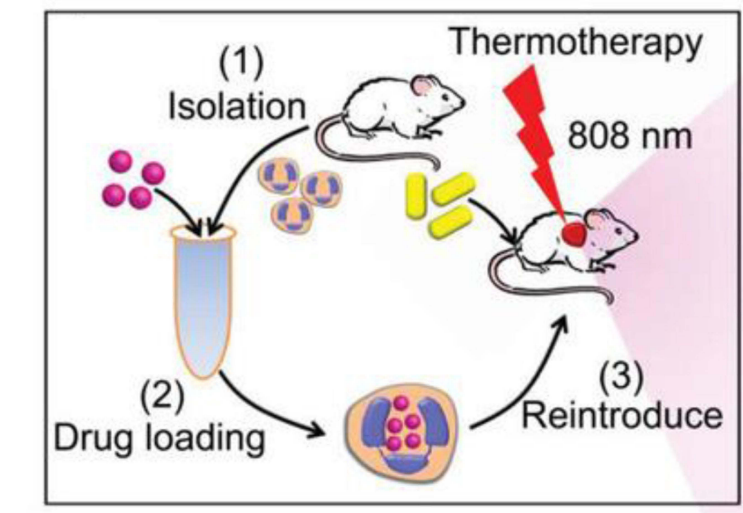

(1) Neutrophil OPTX-CL PEG-GNRs

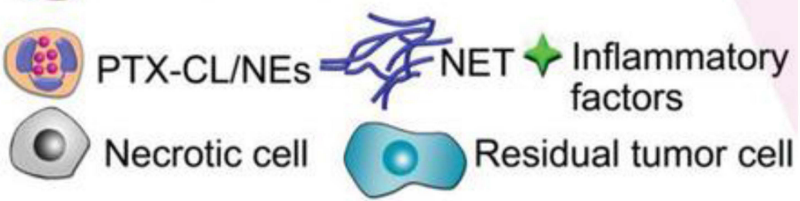

D

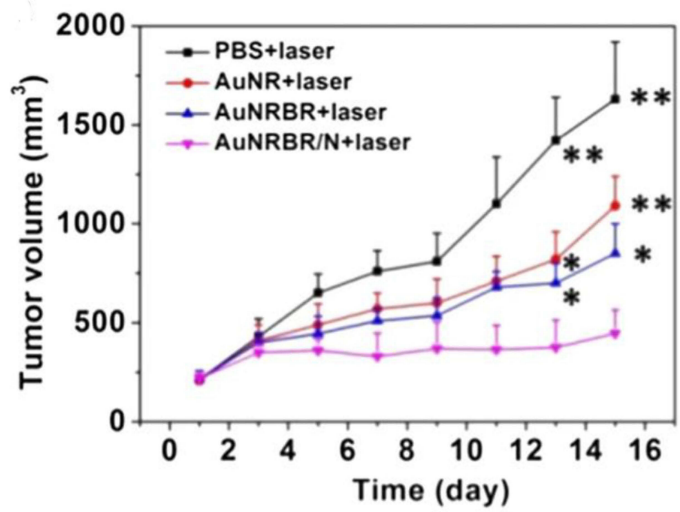

B

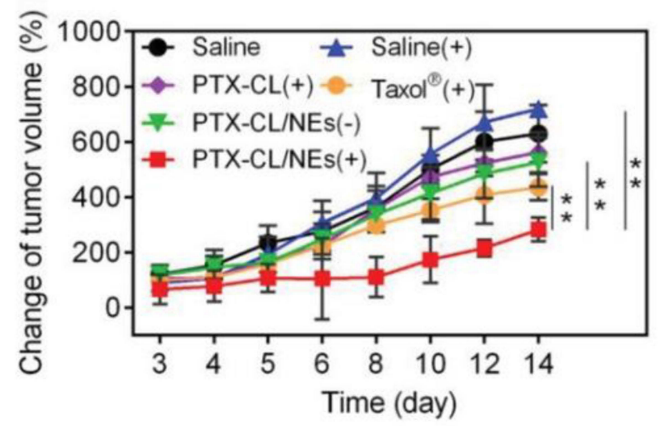

C

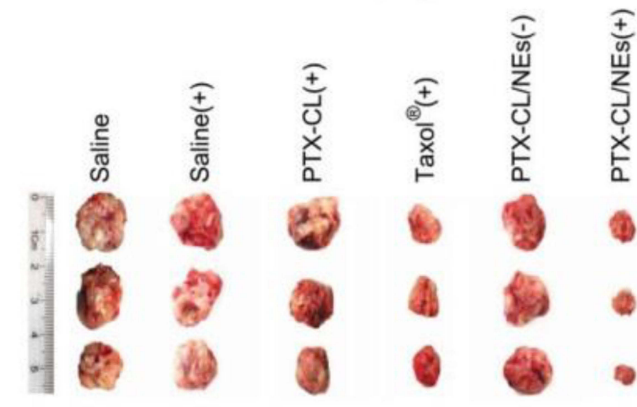

E

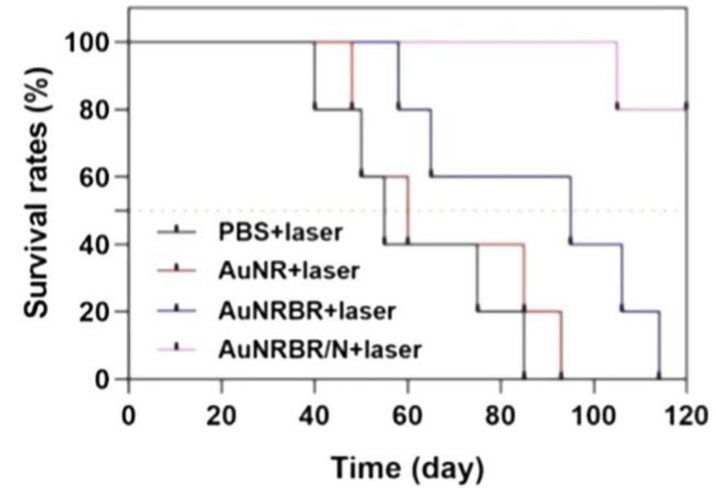

Figure 2 (A) Schematic illustration of PTX-CL/NE preparation and the procedure of administration, including intravenous injection of PEG-GNRs, conduction of PTT, and reintroduction of PTX-CL/NEs. (B) The growth profiles of HepS tumors in mice that received different treatments. The tumor sizes were monitored by a digital caliper, and the tumor volume was calculated according to the following formula: width ${ }^{2} \times$ length $\times 0.5$. (C) Representative pictures of HepS tumors that received different treatments $(n=3)$. Reproduced with permission from Zhang L, Zhang Y, Xue YN, et al. Transforming Weakness into Strength: Photothermal-Therapy-Induced Inflammation Enhanced Cytopharmaceutical Chemotherapy as a Combination Anticancer Treatment. Adv Mater. 2019, 3I, 1805936. (C) 20I8 WILEY-VCH Verlag GmbH \& Co. KGaA, Weinheim. ${ }^{70}$ (D) Tumor growth curve of mice with different treatments during 15 days. (E) Survival curves of Lewis tumor-bearing mice after various treatments. AuNRBR/N injected mice showed improved survival over 120 days after treatments. Reproduced from Ye B, Zhao B, Wang K, et al. Neutrophils mediated multistage nanoparticle delivery for prompting tumor photothermal therapy. J Nanobiotechnol. 2020, 18 (I), I38. Copyright $@ 2020$, The Authors. Creative Commons CC BY. ${ }^{72} * P<0.05$ and $* * P<0.01$.

combination with the monoclonal antibody TA99 and PTT, tumor growth was markedly suppressed (Figure 3D), and the survival time of melanoma-bearing mice was extended (Figure 3E). $^{74}$

Neutrophils in peripheral blood usually highly express CD11b (Mac-1) when activated. Based on this principle, anti-CD11b-coated nanoparticles were synthesized to realize targeting delivery in vivo due to uptake by neutrophils with the assistance of chemotaxis in the microenvironment generated by photosensitizer. Another approach to achieve the goal of hitchhiking was the natural interaction between polysialic acid-octadecylamine (PSA-ODA) and L-selectin on the surface of neutrophils. PSA-ODA was modified onto the surface of the pixantrone (Pix) liposome (Pix-PSL), which was captured efficiently by neutrophils in peripheral blood to deliver to the tumor. Cell uptake proved that the efficiency of Pix-PSL engulfed by neutrophils from the peripheral blood of tumor-bearing mice was significantly higher than that of the control groups, indicating that Pix-PSL was able to effectively target neutrophils. Subsequently, with the S180 and A549 tumor-bearing mice models, the anti-tumor efficacy of Pix was investigated, and the tumor inhibition 

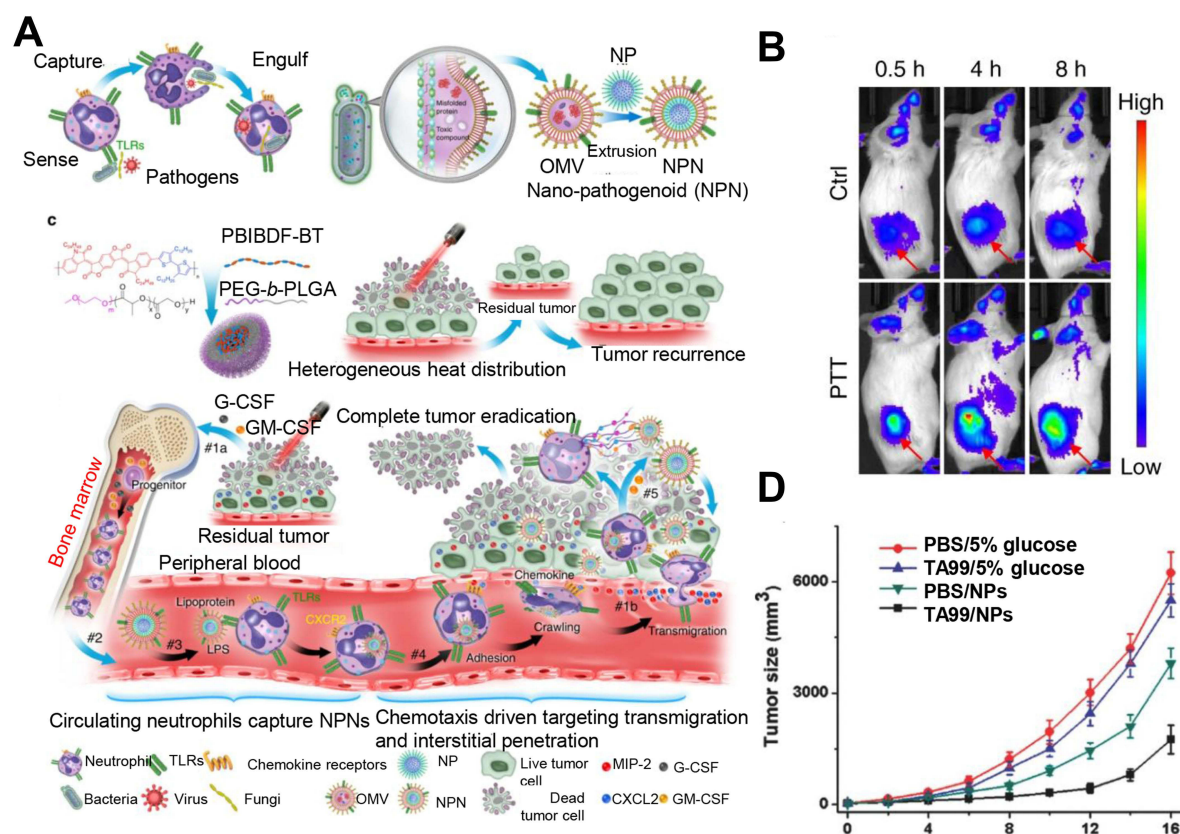

C

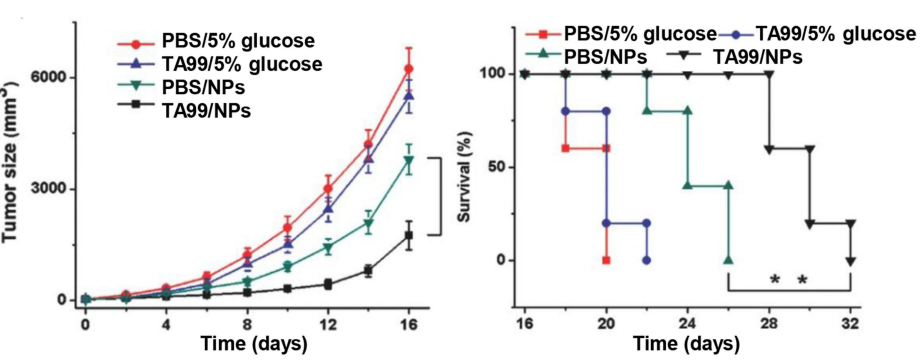

Figure 3 (A) Schematic illustration showing the chemotaxis-driven delivery of NPNs for complete eradication of tumors post-phototherapy. (B) DiD-labeled NPNs were i. v. injected into control or PTT-treated EMT6-bearing mice $(n=3)$. At different time points, in vivo DiD fluorescent signals were observed with IVIS. (C) Two treatments of PTT plus NPNs@Pt completely eradicated tumors in all mice. Tumor growth curves during the treatments. Reproduced from Li M, Li SY, Zhou H, et al. Chemotaxis-driven delivery of nano-pathogenoids for complete eradication of tumors post-phototherapy. Nat Commun. 2020, II (I), I I26. Copyright @ 2020, The Authors. Creative Commons $\mathrm{CC} \mathrm{BY}^{73}$ (D) Tumor size and (E) survival rates of the mice bearing melanoma illuminated with $660 \mathrm{~nm}$ laser at day 2 after the injection of vehicles, TA99, Ppa-loaded NPs, or both of TA99 and Ppa-loaded NPs. The doses of TA99 and Ppa were 40 and $2 \mathrm{mg} \mathrm{kg}^{-1}$, respectively. Reproduced with permission from Chu DF, Zhao Q, Yu J, Zhang FY, Zhang H, Wang ZJ. Nanoparticle Targeting of Neutrophils for Improved Cancer Immunotherapy. Adv Healthc Mater 2016, 5 (9), 1088-1093. @ 2016 WILEY-VCH Verlag GmbH \& Co. KGaA, Weinheim. ${ }^{74} * * P<0.01, * * * P<0.001, * * * * P<0.0001$.

index was used to preliminarily evaluate its efficacy and toxicity. The results showed that Pix-PSL had the best antitumor effect and the lowest toxicity among Pix preparation. ${ }^{75}$

In-situ hitchhiking to neutrophils has been a promising strategy for nanotheranostics, but the protein corona around nanoparticles often plays a negative role in the targeting and accumulation of the tumor. Not desiring to explore the synthesis method to reduce the effect of the corona, a recent study reported an alternative to turning the corona into an advantageous component for the treatment of inflammation in the lung based on the interaction of corona with blood cells. It was found that engineered liposomes with antiphosphocholine lipids can rapidly enrich complement fragment $\mathrm{iC} 3 \mathrm{~b}$ through the effect of voluntary conditions, which were hijacked by neutrophils through the phagocytosis of complement receptor 3. Neutrophil targeting was dependent on the condition of the cells, and only those cells activated by acute inflammation could achieve effective neutrophil targeting. In this study, neutrophils containing liposomes could migrate across the alveolarcapillary barrier, accumulate in the inflamed lung parenchyma within several hours, and release their effective substances to remove bacteria. ${ }^{76}$

\section{Neutrophil Membrane Biomimetic Drug Delivery System}

Targeting the biomimetic cell membrane system plays an active role in dominating cancer therapy, and the integrity of the cell membrane handles positive therapeutic outcomes. An effective approach to maintain the protein integrity of neutrophil membrane was reported. In this study, the innate molecule inhered from mother cells helped to target circulating tumor cells and tumor node metastasis (Figure 4A). The cell membrane was harvested by differential centrifugation, which was coated onto poly (lactic-co-glycolic) acid (PLGA) nanoparticles, showing the structure of the cell membrane with a size of $10 \mathrm{~nm}$ observed by transmission electron microscope (TEM). Membranes extracted from lipopolysaccharide-activated neutrophils were cloaked into PLGA, which could capture circulating tumor cells (CTCs) and metastatic tumor nodes by the interaction between CD44/ICAM-1. After loading the proteasome inhibitor carfezomib, CTCs in the peripheral, blood could be consumed by this biomimetic drug 
delivery, and thus preventing early metastasis (Figure 4B). ${ }^{50}$

Despite neutrophils exhibiting chemotaxis in targeting tumors, researchers have attempted to enhance targeting efficiency by positive feedback. During the therapy, the tumor microenvironment was intervened to trigger amplified targeting signals for a permanent therapeutic effect. Multifunctional two-dimensional nanosheets-black

A Neutrophils-mimicking nanoparticles

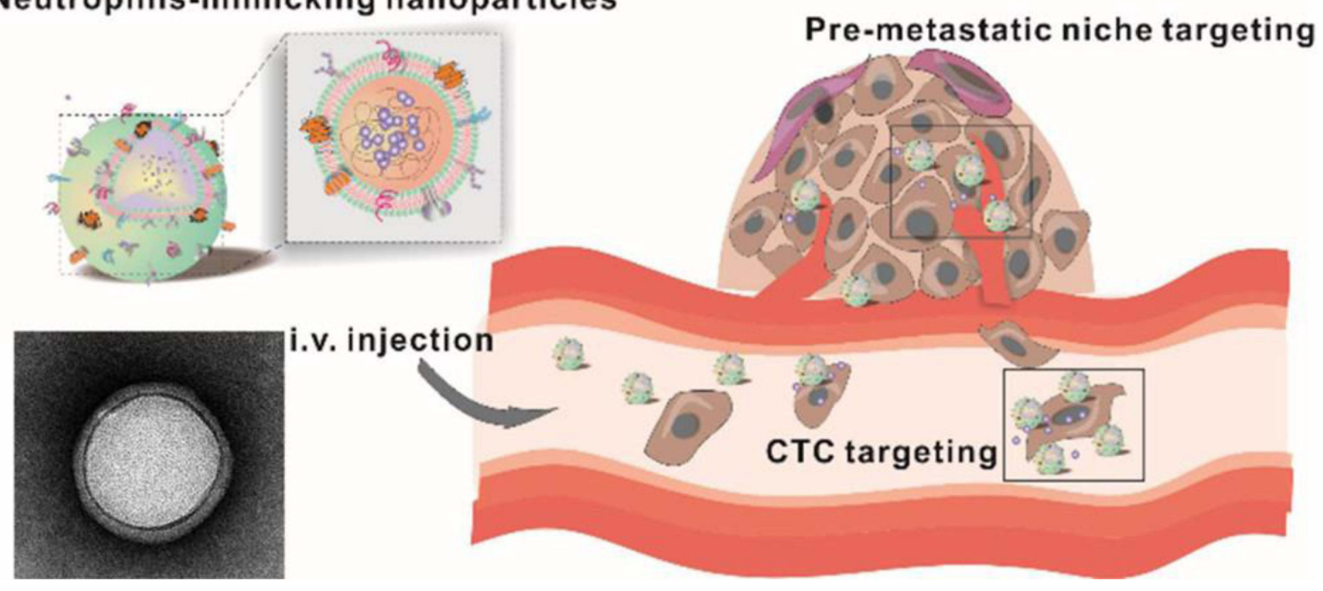

B
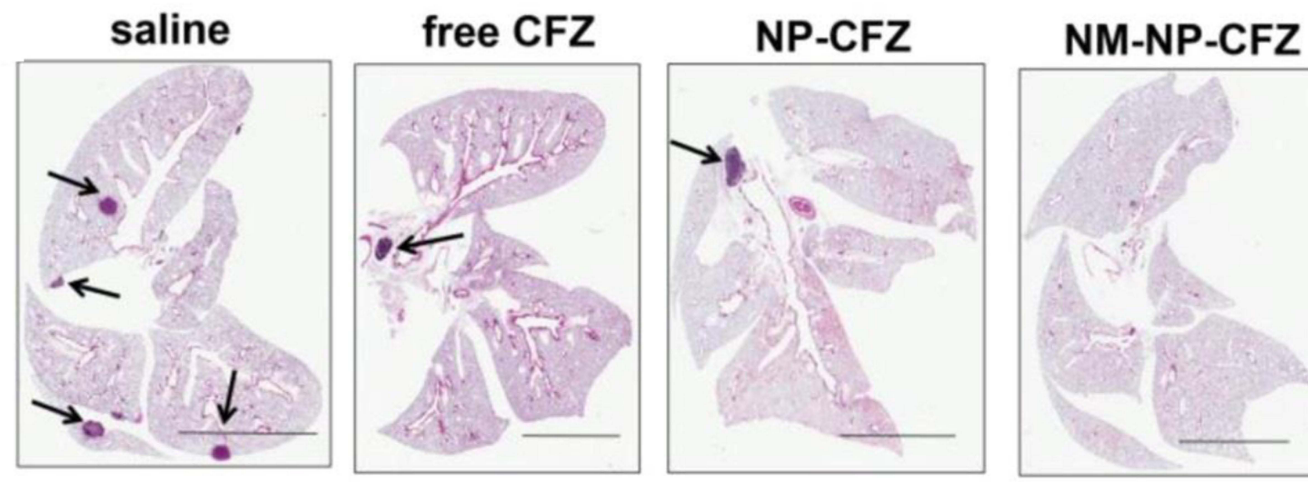

C

D
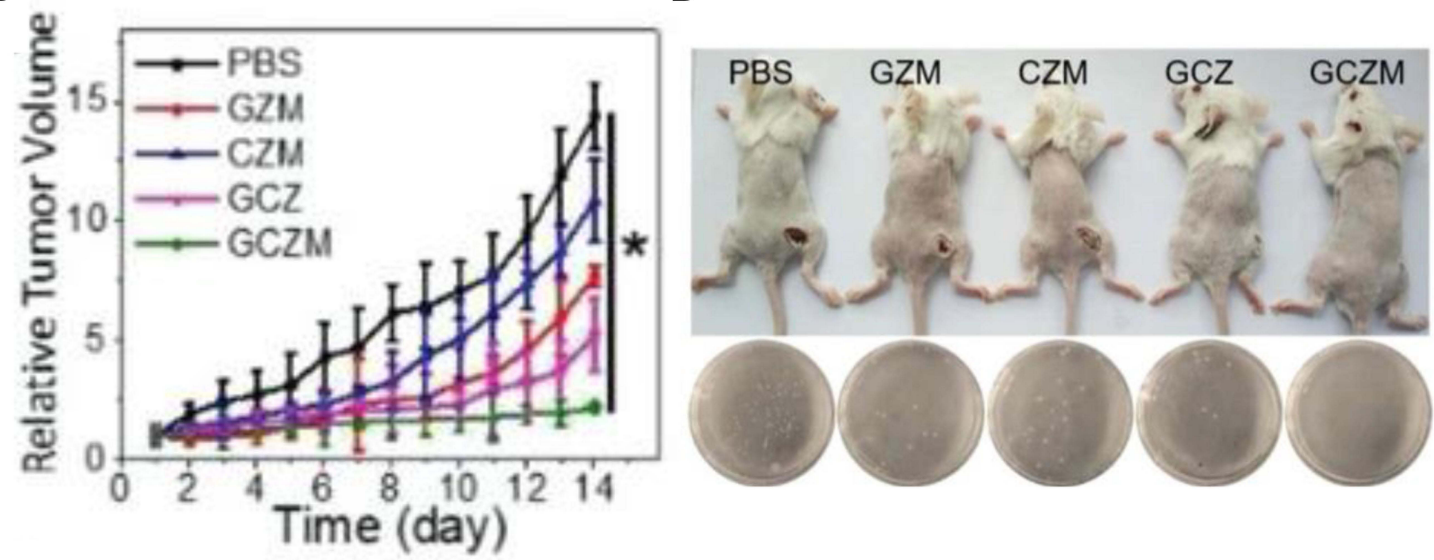

Figure 4 (A) Schematic illustration of NM-NPs loaded with carfilzomib (NM-NP-CFZ) that selectively deplete CTCs and their site of colonization. (B) Histological examination (H\&E staining) of lung tissues after mice were treated with saline and low-CFZ dosage ( $0.5 \mathrm{mg} / \mathrm{kg})$ of free CFZ, NP-CFZ, and NM-NP-CFZ, respectively, on days $0,7,14$, and 21 after inoculation $(n=3)$. Arrows show metastatic nodules. Scale bar, $5 \mathrm{~mm}$. Reproduced with permission from Kang T, Zhu QQ, Wei $D$, et al. Nanoparticles Coated with Neutrophil Membranes Can Effectively Treat Cancer Metastasis. ACS Nano 2017, II (2), 1397-I4II. Copyright @ 2017, American Chemical Society. ${ }^{50}$ (C) Relative tumor volume of 4TI-tumor-bearing mice. (D) Representative images of mice and S. aureus colonies on LB agar plates of S. aureus-infected mice at the 7th day. Reproduced with permission from Zhang C, Zhang L, Wu W, et al. Artificial Super Neutrophils for Inflammation Targeting and HClO Generation against Tumors and Infections. Adv Mater 2019, 31 (19), el90I I79. (c) 2019 WILEY-VCH Verlag GmbH \& Co. KGaA, Weinheim. ${ }^{80} * P<0.05$. 
phosphorus (BP) conjugated with transforming growth factor- $\beta$ inhibitor was coated with neutrophil membrane. BP mediated photothermal therapy (PDT)/PTT could induce local acute inflammation in tumors. At the same time, the inflammatory tendency of the neutrophil membrane could promote the accumulation of nanoparticles in the tumor site after subsequent administration. The results showed that PDT/PTT combined with immunotherapy could destroy local primary tumors and inhibit remote metastases, alleviating the problem of undesired treatment of metastatic malignant tumors. ${ }^{77}$

Apart from targeting, the cell membrane inherited another specific functionality-permeability, which endowed biomimetic nanoparticles with the ability to bypass the barrier in the body. Due to the main relationship between inflammation and cancer, celastrol, which has anti-inflammation activity, was used for cancer therapy. After celastrol was loaded with PEG-PLGA, neutrophil membranes were further coated to treat pancreatic cancer. It was illustrated that the blood pancreatic barrier could be surmounted, and that precise drug delivery to the pancreas could also be realized. In the tumor-bearing mice model, membrane cloaking nanoparticles displayed more accumulation in the tumor site compared to bare nanoparticles after administration. In the local or ectopic tumorbearing mice model, biomimetic nanoparticles showed increased tumor suppression, and the survival rate was extended with reduced hepatic metastases. ${ }^{78}$

Although the above-mentioned nanoparticle delivery systems based on neutrophil membranes have shown the advantage of targeting tumors, the endocytosed efficiency was low due to the absence of specific interactions between neutrophil-membrane-coated nanoparticles and cells. As a result, it is still imperative to enhance the efficiency of endocytosis to further improve tumor treatment. Neutrophil membrane camouflaging nanoparticles (TNM-PN) modified with a tumor necrosis factor-related apoptosis-inducing ligand loaded with the drug PTX were conducive to cell internalization. In this research, prolonged blood circulation to $48 \mathrm{~h}$ and preferential tumor accumulation in mice in the TNM-PN group were achieved significantly, with obvious tumor suppression and survival rates. The outcome of tumor treatment was attributed to targeting adhesion from neutrophil membranes originating from immune escape and subsequent excellent cell internalization. ${ }^{79}$

As a derivative of powerful effector leukocytes, the biomimetic neutrophil membrane system has the ability to simultaneously target at tumor. A group designed an artificial super neutrophil with good inflammation and tumor targeting, as well as the ability to generate hypochlorous acid ( $\mathrm{HClO})$, which can be used to target and eliminate pathogens. Glucose oxidase and chloroperoxidase were embedded in $\mathrm{ZIF}-8$ to generate $\mathrm{HClO}$ and wrapped with neutrophil membranes to fabricate the super neutrophils. The results showed that synthetic super neutrophils were seven times more effective in eliminating tumors and infections than natural neutrophils, suggesting that super neutrophils have great value and potential in biomedical application (Figure 4C and D). ${ }^{80}$

\section{Neutrophil Exosome-Inspired Drug Delivery Systems}

Exosomes inherited certain functionalities of source cells in crossing the blood-brain barrier and targeting inflammation, which, as a consequence, could be used as a delivery system for glioma. Through the establishment of bloodbrain barrier models in vitro and in vivo, the neutrophil exosome-based drug delivery system (NEs-Exos) was systematically verified with the effect of passing through the blood-brain barrier, inflammatory chemotactic, and glioma killing by inflammation and glioma models. The results showed that NEs-Exos had significant characteristics with the exosome membrane protein. Compared with free DOX and exosomes originating from red blood cell, NEs-Exos /DOX could respond to the inflammatory chemokine fMLP and had similar inflammatory chemotactic properties to the source cell, which was proved both by confocal laser imaging and DOX quantitative analysis in the lower chamber of the in vitro blood-brain barrier model. Evaluation of the blood-brain barrier through the zebrafish model (transgenic zebrafish, green fluorescent proteinlabeled blood vessels) showed that NEs-Exos/DOX could successfully penetrate the blood-brain barrier to reach the brain parenchyma. Within $24 \mathrm{~h}$, an obviously increased fluorescence signal was detected in the brain tumors of mice treated with NEs-Exos/DiR comparing with free DiR. The results of immunofluorescence of brain slices confirmed that NEs-Exos significantly accumulated in the area of inflammation and tumor in-situ administration and further pointed out that the NEs-Exos (Dir marked) aggregation area was highly consistent with the tumor tissue area. In contrast with the other groups, a weaker fluorescence signal was observed in the NEs-Exos/DOX group, with obvious suppression of glioma growth. The above results fully underpinned the idea that NEs-Exos could break through the physiological barrier, migrate to the 
inflammatory microenvironment of tumor tissue, and carry drugs into the brain. The pharmacokinetics evaluation results of the orthotopic transplantation model of glioma showed that NEs-Exos/DOX inhibited the rapid growth of glioma compared with the control group and the free DOX group. Importantly, the research fully warranted that NEsExos/DOX had inflammatory response characteristics that could detour the physiological barrier to target and gather in the inflammatory microenvironment of tumors, with an improved anti-glioma effect. This research would not only help provide new drug delivery strategies for the treatment of glioma, but also provide new opportunities for noninvasive targeted drug delivery for other central nervous system diseases. ${ }^{81}$

\section{Neutrophil-Derived Drug Delivery Systems in the Treatments of Inflammation \\ Neutrophil Loading Drug Delivery System}

Despite the development of various anti-bacteria materials and therapies, eliminating infection by bacteria is still a huge challenge, especially for drug-resistant bacteria. Inspired by the recruitment by macrophages that recognize neutrophil apoptosis, neutrophils loading nanoparticles with the property of photocatalysis were leveraged to activate the immune system to generate anti-bacteria response. Neutrophils with photocatalytic nanoparticles reserved the entire functionality of the source cell. When accumulating in the infected area, photocatalytic nanoparticles loaded neutrophils could induce apoptosis along with the release of ROS leading to the secretion of chemokines for recruiting macrophages in the infected area. In the infectious mice model built by pathogenic bacteria, low-dose photocatalytic nanoparticle-laden neutrophils displayed pronounced therapeutic outcomes by boosting macrophage recruitment and a cascaded immune response. $^{82}$

In addition to photo-induced neutrophil apoptosis, a visible alternative to curb neutrophil apoptosis is by employing chemotherapeutic drug. Apoptosis is programmed cell death to maintain immune homeostasis, but inflammation or tissue damage destroys the neutrophil death program, leading to many diseases. Precise control of neutrophil apoptosis can remove inflammation and restore immune homeostasis. It was reported that DOXconjugated protein nanoparticles could selectively target inflammatory neutrophils in-situ and deliver DOX into the cells to induce apoptosis (Figure 5A). The release of DOX was triggered by the acidic environment in neutrophils (Figure 5B), leading to an increased neutrophil apoptosis rate induced by DOX-hyd-BSA NPs, which subsequently prevented migration to inflammation (Figure $5 \mathrm{C}$ ). In the established middle cerebral artery occlusion (MCAO) mouse model to mimic cerebral ischemia/reperfusion (Figure 5D), myeloperoxidase (MPO), a marker of neutrophils, was observed to reduce obviously, implying that the inhibition of neutrophils was conductive in preventing brain damage during cerebral ischemia/reperfusion, but not suppressing systemic immunity thereafter (Figure $5 \mathrm{E}$ ). ${ }^{83}$

The unrestricted migration of neutrophils to the cerebral ischemic area and the subsequent release of ROS are believed to be responsible for reperfusion injury after acute ischemic stroke (AIS). Therefore, reducing the infiltration of inflammatory neutrophils may be the desired treatment for AIS. Inspired by specific cell recognition between platelets and inflammatory neutrophils, platelet mimicking nanoparticles (PTNPs) were used to directly identify, intervene, and monitor inflammatory neutrophils in AIS treatment. After PTNPs were loaded with piceatannol and superparamagnetic iron oxide (SPIO), the adherent neutrophils could be successfully identified due to the platelet membrane coating. The loaded piceatannol could be internalized by the attached neutrophils and detached during circulation, thereby reducing the infiltration of neutrophils and the infarct area (Figure 5F). When combined with magnetic resonance imaging, internalized SPIO could be used to monitor inflammatory neutrophils in real time, which correlated with the therapeutic effect (Figure $5 \mathrm{G}$ and $\mathrm{H}$ ). ${ }^{84}$

\section{Neutrophil Membrane Biomimetic Drug Delivery System}

Rheumatoid arthritis (RA) is a chronic disease experienced worldwide and is the prime factor responsible for human disability. Currently, the therapy for RA is not satisfactory due to the diversity and complex interaction of cytokines used in clinics. A chimeric strategy employing neutrophilmembrane-coated nanoparticles was exploited for the treatment of RA. In this biomimetic nanoparticle, the neutrophil membrane on the surface inherited the functionality of mother cells with the relative antigen and membrane structure, which set a natural trap for biomolecules targeting neutrophils. Biomimetic nanoparticles could neutralize proinflammatory cytokines to suppress inflammation and penetrate the cartilage matrix to provide powerful cartilage protection to prevent joint damage. The enhanced penetration may lie in 
A

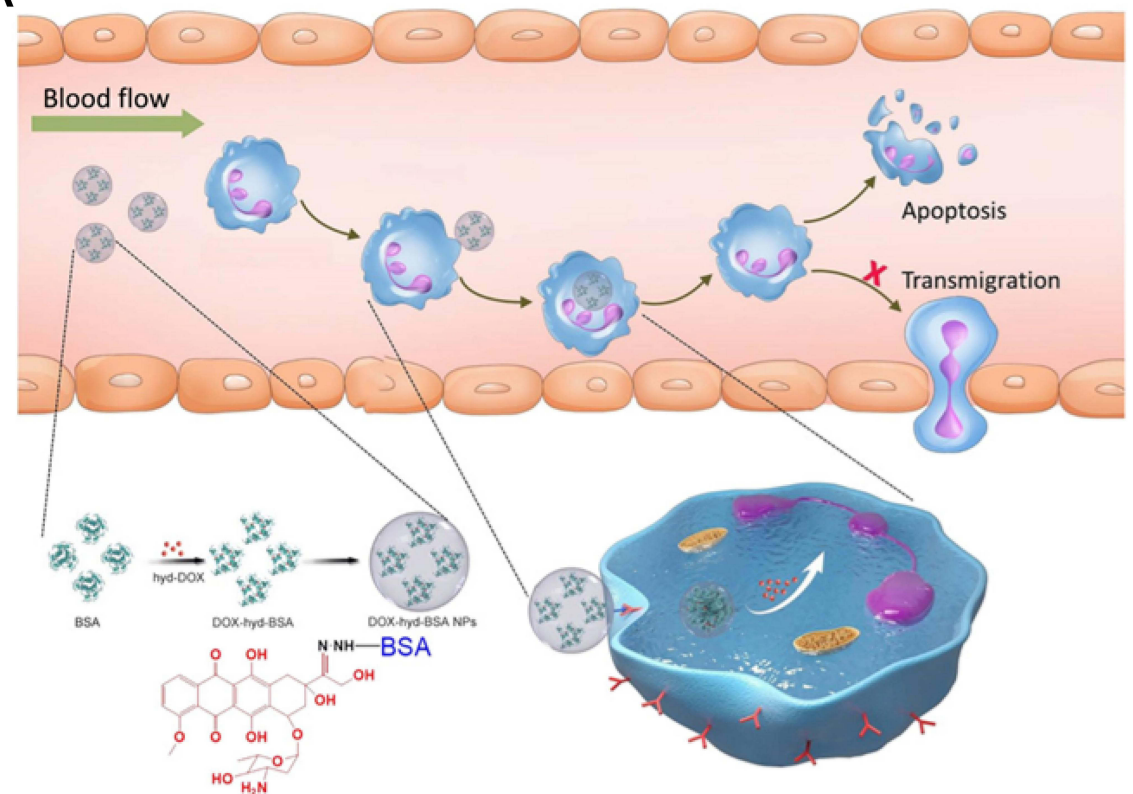

D

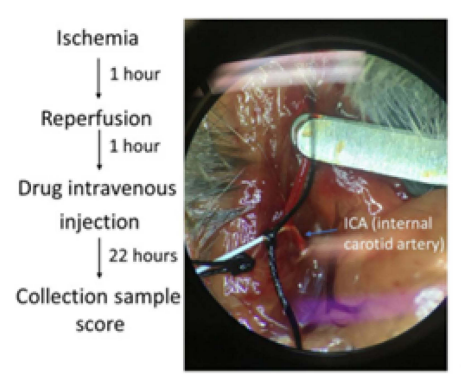

E

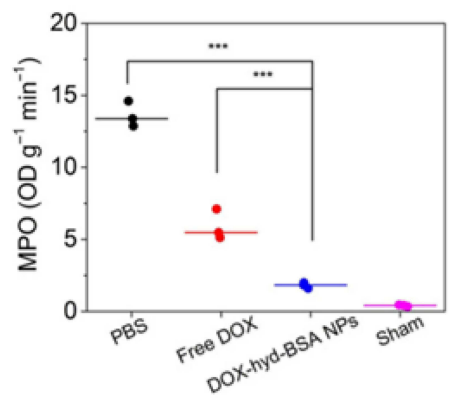

B

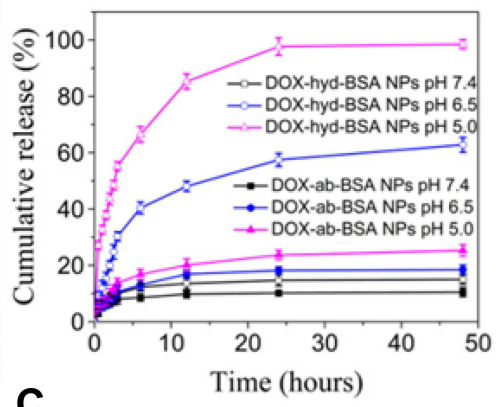

$c$

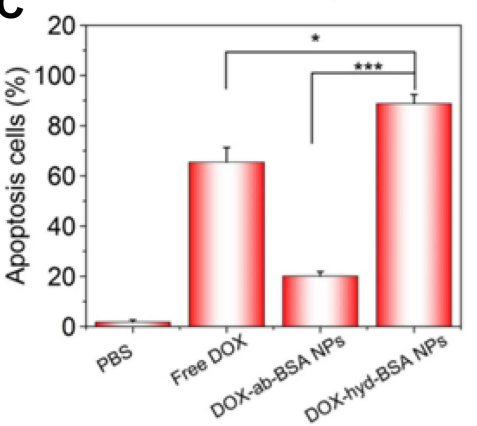

F

Sham

Saline

TNP

PTNP

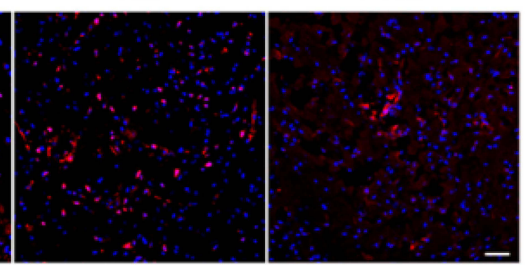

G

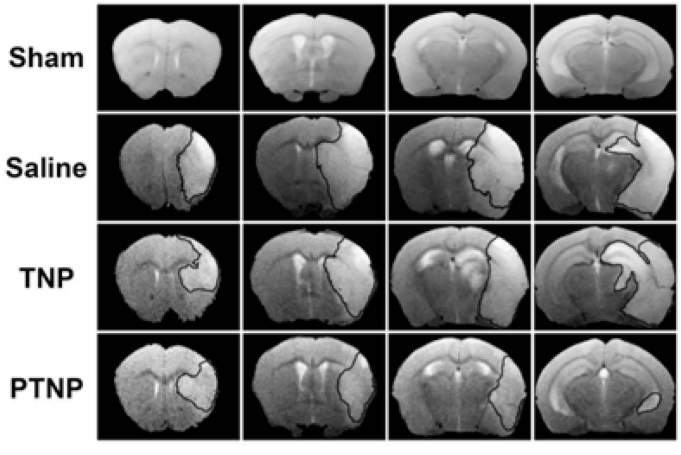

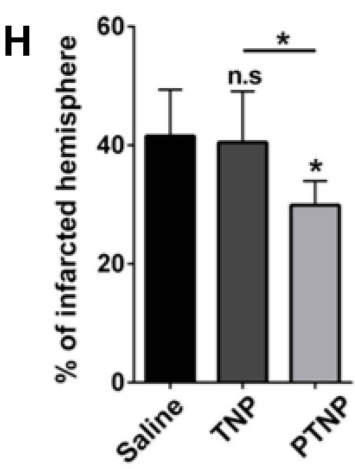

Figure 5 (A) Scheme showing NP targeting of proinflammatory neutrophils to induce their apoptosis for treatment of inflammatory diseases. (B) Cumulative DOX release from DOX-ab-BSA NPs or DOX-hyd-BSA NPs in PBS at pH 7.4, 6.5, or 5.0. (C) Percentage of apoptotic cells analyzed by flow cytometry after HL-60 cells treated with various DOX formulations. (D) Experimental design to examine the benefit of DOX-hyd-BSA NPs in cerebral I/R. OD, optical density. (E) MPO activity in brain damage tissues at $22 \mathrm{~h}$ after administration of PBS, free DOX, and DOX-hyd-BSA NPs. Reproduced with permission from Zhang, CY, Dong, XY, Gao, J, Lin, WJ, Liu, Z, Wang, ZJ, Nanoparticle-induced neutrophil apoptosis increases survival in sepsis and alleviates neurological damage in stroke. Sci Adv 2019,5 (II), eaax7964. Copyright @ 20I9 The Authors. Creative Commons Attribution Non Commercial License 4.0 (CC BY-NC) ${ }^{83}$ (F) CLSM images of neutrophil infiltration in ischemic brain regions after treatment with saline, TNPs, and PTNPs. Neutrophils were immunostained with anti-Ly6g antibody (red) and nuclei were stained using DAPI (blue). Scale bar: $50 \mu$ m. (G) T2-weighted images of ischemic brains at $24 \mathrm{~h}$ in tMCAO mice treated with saline, TNPs, and PTNPs. The black curve indicates the infarct region. $(\mathbf{H}) \mathrm{Quantification}$ of the infarction volumes in saline-, TNP-, and PTNP-treated tMCAO mice. Error bars indicate SD $(n=5)$. Reproduced with permission from Tang CM, Wang C, Zhang Y, et al. Recognition, Intervention, and Monitoring of Neutrophils in Acute Ischemic Stroke. Nano Lett 2019, 19 (7), 4470-4477. Copyright $\mu 2019$ American Chemical Society. ${ }^{84} * \mathrm{P}<0.05$, and $* * * \mathrm{P}<0.001$.

the adhesion between neutrophils and cartilage cells. In the transgenic mice model and the mice induced by collagen, biomimetic nanoparticles could improve joint damage and alleviate the severity. Although neutrophils-membranecoated nanoparticles were locally injected and retained primarily at the knee joint, invasive inflammatory factors could 
be eliminated to trigger a systemic response to reduce the progression and severity of RA. ${ }^{49}$

Acute pancreatitis (AP), as inflammation, makes substantial patients afflicted with a painful feeling. It has been demonstrated that PEG-PLGA loading celastrol (CLT) was coated with neutrophils membrane (NNPs/CLT) for the treatment of AP (Figure 6A). The biomimetic nanoparticles could overcome the blood pancreatic barrier, accumulating selectively in the pancreas of rats with AP (Figure 6B). Compared with bar nanoparticles loading CLT, NNPs/CLT downregulated the levels of serum amylase and pancreatic myeloperoxidase levels in rats with AP. Moreover, the utilization of neutrophil membrane camouflaging nanoparticles significantly reduced the systemic toxicity of CLT to rats. ${ }^{85}$

As for anti-infection therapy, there still exists a challenge in precisely releasing metal ions against infection. Fortunately, biomimetic system could handle this problem by near-infrared (NIR)-mediated PDT to curb the condition of oxidation to realize the on-demand activation and controlled release of metal ions. Ag nanoparticles (AgNPs) were decorated onto a porous coordination network of porphyrin (PCN), followed by modification with neutrophil membranes (PAM). Under NIR irradiation of the infected tissue, the biomimetic nanomaterials generated singlet oxygen locally, partially degrading the AgNPs and releasing cytotoxic $\mathrm{Ag}^{+}$for metal ion therapy. Meanwhile, the introduced AgNPs promoted the singlet oxygen production rate of $\mathrm{PCN}$ due to the local electric field effect. Therefore, the NIR-controlled interaction between AgNPs and PCN may provide huge potential for realizing controllable, precise, and effective disease treatment with reduced side effects. ${ }^{86}$

Neuroinflammation, which plays a key role in strokeinduced brain injury, is a promising target for stroke treatment. However, there is still a lack of a satisfactory nanoprobe to selectively monitor neuroinflammation, which is an important mode to guide specific treatment. Inspired by the interaction of neutrophils and endothelium, neutrophil disguised magnetic nanoprobes (NMNPs) were designed, which can be used to target activated endothelial cells for improved neuroinflammation imaging. NMNPs were composed of superparamagnetic iron oxide (SPIO) loaded with PLGA nanoparticles, followed by coating with a biomimetic neutrophil membrane. Importantly, in vivo imaging of the brain microvasculature in mice with transient middle cerebral artery occlusion (tMCAO) was carried out to prove that NMNPs could successfully bind to the inflamed cerebrovascular system. Besides, NMNPs further accumulating in the cerebrovascular system was

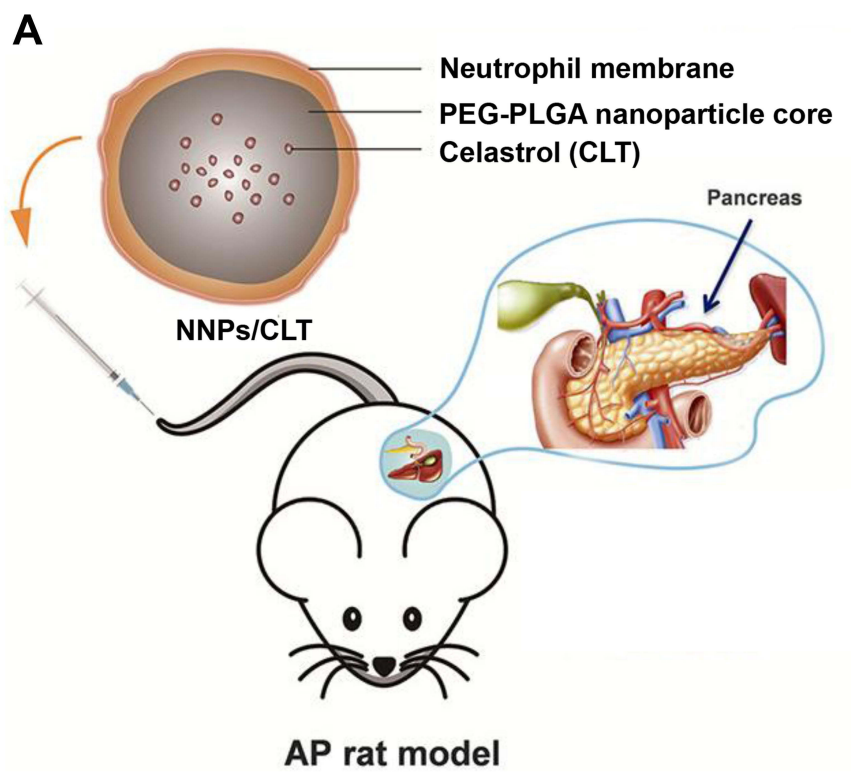

B

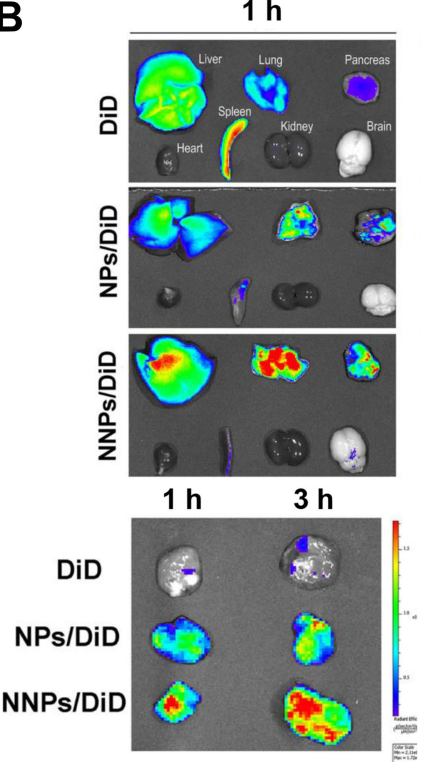

$3 \mathbf{h}$

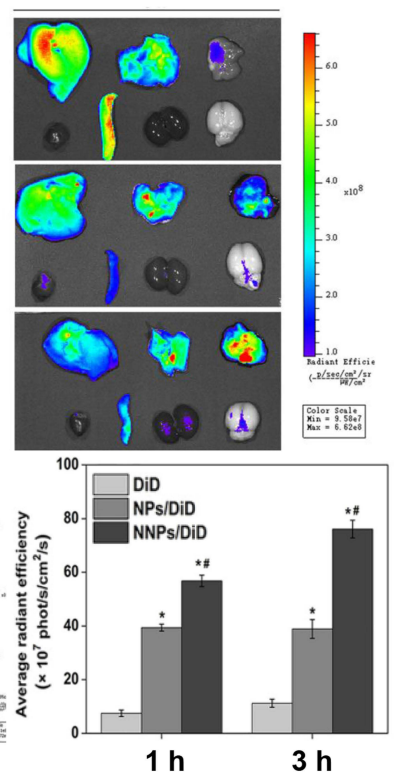

Figure 6 (A) Schematic representation of Celastrol (CLT)-loaded PEG-PLGA nanoparticles (NPs/CLT) coated with neutrophil membranes (NNPs/CLT) for the management of acute pancreatitis (AP). (B) NNPs/DiD selectively accumulated in inflamed pancreas. Ex vivo fluorescent imaging of major organs in AP rats at I and $3 \mathrm{~h}$. Ex vivo fluorescent imaging of pancreas in AP rats at I and $3 \mathrm{~h}$. Semiquantitative analysis of fluorescent intensity within the pancreas. Reproduced with permission from Zhou $X$, Cao X, Tu H, Zhang ZR, Deng L. Inflammation-Targeted Delivery of Celastrol via Neutrophil Membrane-Coated Nanoparticles in the Management of Acute Pancreatitis. Mol Pharmaceut 2019, 16 (3), 1397-1405. Copyright (C) 2019 American Chemical Society. ${ }^{85} * P<0.05$ and ${ }^{\#} P<0.05$. 
verified, suggesting a good contrast effect in neuroinflammation and biosafety induced by stroke. ${ }^{87}$

\section{Neutrophil Exosome-Inspired Drug Delivery Systems} Recent research has unveiled a new strategy by employing neutrophil exosomes, that is, the utilization of nanodelivery to successfully treat bacterial growth and inflammation in the lungs of mice, including COVID-19. Resolvins belong to mediators that are specially produced by omega- 3 fatty acids during the acute inflammatory response to infection or tissue damage. Lung infections usually lead to two problems: infection and inflammation caused by a strong immune system response. As a result, the researchers used two drugs to treat this bottleneck. In this study, RvD1 and antibiotics (ceftazidime) were packaged into neutrophil exosomes specifically targeting inflammation of the lungs (Figure 7A). Compared with healthy mice, the neutrophil exosome loading drug delivery system exhibited increased accumulation in the lungs under the challenge of lipopolysaccharide (LPS). The quantitative analysis by IVIS images suggested that the accumulation increased fivefold compared to the controls. Neutrophil exosomes had long circulation in healthy mice in the pharmacokinetics study, and LPS-induced lung inflammation increased the deposition of exosomes in the lung (Figure 7B), indicating that these exosomes could specifically target the inflamed vasculature for the treatment of lungs infected by Pseudomonas aeruginosa. The research provides evidence that this innovative method could effectively fight lung inflammation, and various medicines could be loaded into this system to treat a range of infectious diseases. ${ }^{88}$ The delivery system was further used to deliver two drugs for the treatment of peritonitis. RvD1 was inserted on the surface of neutrophil exosomes, as well as ceftazidime (CEF) loaded inside, which could improve the infection caused by bacteria. In the mice model of bacterial peritonitis, exosomes helped the codelivery of RvD1 and CEF so that the inflammation was reduced and bacteria was killed (Figure 7C). The results showed that neutrophil exosomes were crucial for enhancing the treatment of infectious disease. ${ }^{89}$ Additionally, in the pathological mechanism of ischemia reperfusion, neutrophils adhered to blood vessel endothelial cells to trigger inflammation response. Based on this, resolving-RvD2-loaded neutrophil exosomes were exploited to fight with inflammation for the protection of brain damage in ischemic stroke. The system could obviously reduce inflammation of ischemic stroke and improve the neurological function of mice (Figure 7D).$^{90}$ Systemic sclerosis (SSc) belongs to autoimmune disease with the feature of vasculopathy of several organs, inflammation, and fibrosis. Neutrophil exosomes containing various DNA, RNA, and proteins play a key role in SSc. It was reported exosome isolated from peripheral blood mononuclear cells or neutrophils have been reported to suppress the proliferation and migration of HDMEC. ${ }^{39}$

\section{The Future Prospects}

The pathogenesis of most diseases is related to innate and adaptive immune responses, in which neutrophils play an important role. Drug delivery involves several stepsincluding circulation, accumulation, deep penetration, endocytosis, and drug release. Compared with traditional nanotheranostics, a bioinspired system based on neutrophils can significantly improve systemic circulation and targeting efficiency along with the improved treatment of inflammation and tumors. However, there are still some concerns that need to be addressed in the precise delivery of drugs to the target, such as how to realize deep penetration and efficient endocytosis. To improve the efficiency of drug delivery, it is vital to reasonably design each step during this process. Therefore, designing nanocarriers with appropriate size, shape, and surface chemical properties, the selection of suitable drugs to be carried by neutrophils in the nanotheranostics system, and the control of the release of nanoparticles in the disease site are crucial factors impairing the advantages of neutrophil-based drug delivery systems. Furthermore, nanoparticles and neutrophils are easily labeled, allowing for the use of imaging systems to monitor neutrophil-nanoparticle complexes in vivo for the diagnosis of inflammation or tumors in targeting disease sites. Currently, molecular imaging has been used in theranostics, including molecular imaging probes and multifunctional nanomaterials, enabling the visualization of neutrophilbased nanotherapeutic processes.

The proportion of neutrophils in mice is only $10-25 \%$. As the most abundant immune cells in the human body, neutrophils account for $50-70 \%$ of white blood cells, which means that the results obtained from experiments in rats or mice may be more significant when extrapolated to patients. ${ }^{3}$ Neutrophil-based drug delivery and reinfusion strategy is like CAR-T therapy, providing a novel idea for tumor treatment. However, its feasibility and safety still need to be completely verified with respect to clinical transformation. Neutrophils belong to a short-lived cell with an average lifespan of 5.4 days in blood 

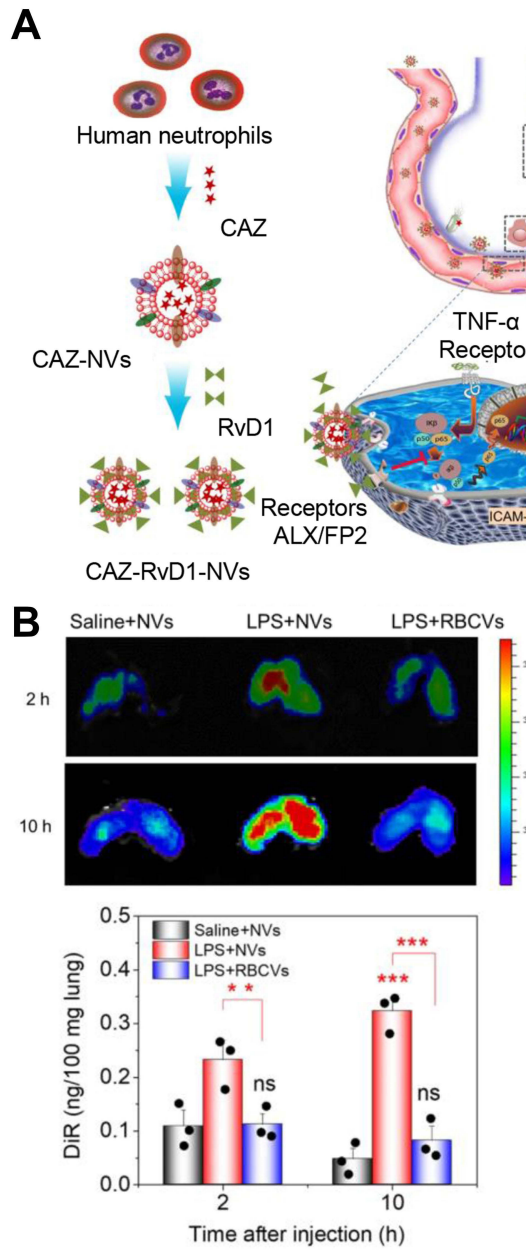

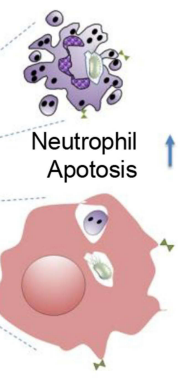

Macrophage $\uparrow$ Phagocytosis

lial Adhesion Molecules

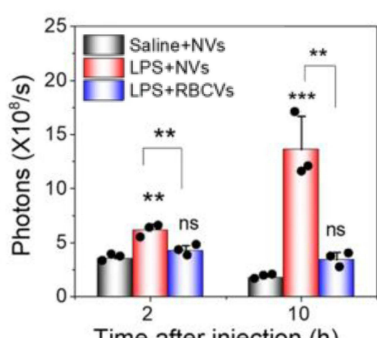

Time after injection (h)

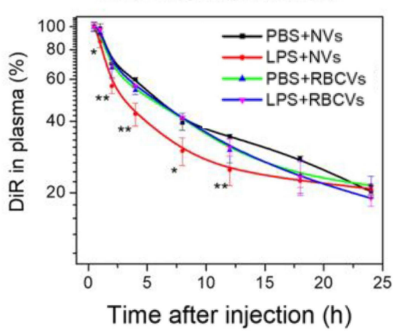

C
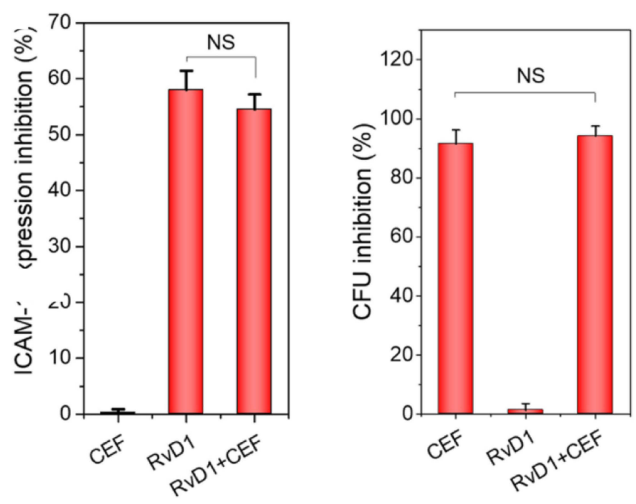

D
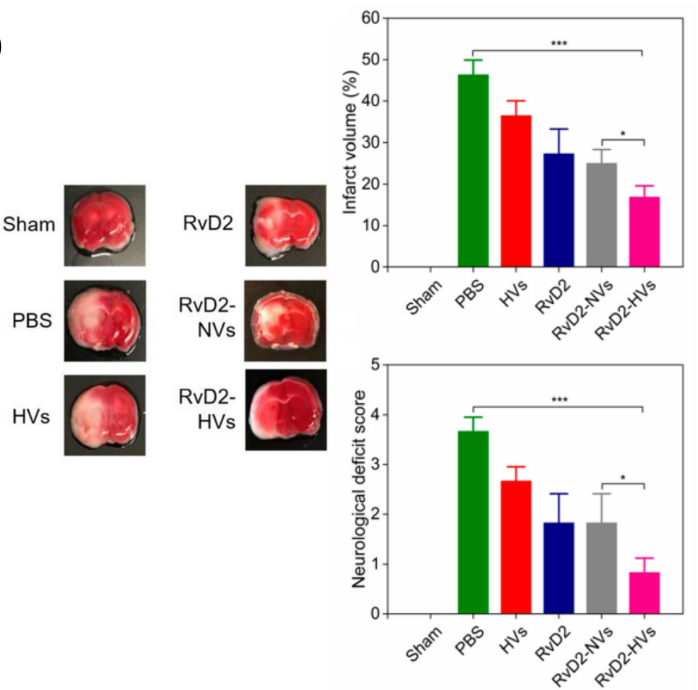

Figure 7 (A) Schematic on development of human neutrophil membrane-derived nanovesicles to target the infectious lung. (B) IVIS images of mouse lungs after LPS (at 5 $\mathrm{mg} / \mathrm{kg}$ ) was intratracheally administered to the mice. Quantitative analysis of fluorescent intensity of lung tissues based on the IVIS images. Nanovesicle fluorescence of lung homogenates was measured at $780 \mathrm{~nm}$. Pharmacokinetics of NVs and or RBCVs in healthy mice or inflamed mice induced by intratracheal LPS administration. Reproduced with permission from Gao J, Wang S, Dong X, Leanse LG, Dai T, Wang Z. Co-delivery of resolvin DI and antibiotics with nanovesicles to lungs resolves inflammation and clears bacteria in mice. Commun Biol 2020, 3 (I), 680. Copyright (C) 2020, The Authors. Creative Commons CC BY. ${ }^{88}$ (C) RvDI and CEF inhibits ICAM-I expression of endothelial cells and bacterial growth, respectively. (D) RvD2-nanovesicles reduce cerebral infarct sizes and protect neurological damage from ischemic stroke at 22 $\mathrm{h}$ postinjection of therapeutics ( $24 \mathrm{~h}$ after the MCAO surgery). Representative mouse brain sections stained with I\% of TTC in sham mice, and MCAO mice treated with PBS, HVs, RvD2, RvD2-NVs, and RvD2-HVs, respectively. Reproduced with permission from Dong XY, Gao J, Zhang CY, Hayworth C, Frank M, Wang Z. J. Neutrophil Membrane-Derived Nanovesicles Alleviate Inflammation To Protect Mouse Brain Injury from Ischemic Stroke. ACS Nano 2019,13 (2), I272-1283. Copyright (C) 20I9 American Chemical Society. ${ }^{90} * P<0.05, * * P<0.01$, $* * * P<0.001$. The ns denotes nonsignificant difference.

circulation. ${ }^{3}$ After being isolated from the blood, neutrophils require purification, drug loading, and reinfusion within a few hours. Additionally, the activity of neutrophils should be guaranteed during the separation process, because once the structure of isolated neutrophils is damaged, the signals of "find-me" or "eat me" will be expressed to trigger phagocytosis by immune cells, resulting in severe off-target. Therefore, to achieve the validity of in vitro neutrophil drug delivery after reinfusion, it is necessary to master the mature separation and drug delivery technology of neutrophils, as well as establish a reliable method for neutrophil activity detection before reinfusion. Finally, the source of many neutrophils must be solved. If patients are administered their own neutrophils, it is necessary to consider the heterogeneity between neutrophils in patients and in healthy humans. Besides, whether neutrophils isolated from different patients can be applied to this therapy still needs to be considered to establish neutrophil classification opportune for drug delivery and reinfusion strategy. If allogeneic neutrophil therapy is used, the safety issues caused by immune rejection after reinfusion should be taken into consideration. Although it was proved that all indicators were normal after human neutrophils were injected back into nude mice 
classified as an animal model of immunodeficiency, the safety of neutrophil transfusion should at least be reproduced in animal models with robust immunity, or even in humans.

Neutrophils are expected to be used in drug delivery due to their flexible appearance and ability to cross the vascular barrier into diseased tissues. However, the flexibility and mobility of biomimetic nanomaterials originating from neutrophils are far lower than those of neutrophils. Existing technologies can successfully realize the isolation and extraction of neutrophil membranes to coat on the surface of nanoparticles, but there is still a lack of characterization of neutrophils-membrane-coated nanodrugs. The current techniques are only limited to the macroscopic characterization of biomimetic systems, including the observation of the nanoparticle morphology by TEM, as well as the detection of the co-location signal between the core of nanoparticles and the fluorescence labeling cell membrane, while the microscopic characterization of the molecular structure on the surface of the cell membrane needs to be underlined. In addition, macrophages are recruited to the vicinity of apoptotic cells, which are phagocytized by immune cells through phosphatidylserine, an "eat me" signal on the outer membrane of apoptotic cells. In the process of preparing biomimetic nanoparticles, ultrasonic dispersion technology will generate local high temperature and metal debris, which may destroy the structure of protein on cell membrane. The redistribution of membrane components caused by extrusion further increases the probability of phosphatidylserine ectropion, adding to the difficulty of maintaining membrane integrity and consistent membrane composition with original cell. If the damaged cell membrane is coated on the surface of the nanoparticles, the biomimetic nanoparticles will be quickly recognized and cleared by the phagocytes in the body, leading to severe off-target and failure to exert the expected therapeutic effect. Maintaining the integrity of the molecular structure on the surface of the cell membrane is crucial to show the prevalence of biomimetic neutrophil-membrane-coated nanoparticles. Otherwise, wearing a damaged coat on the nanoparticles will not only fail to endow drug carriers with the intended role but will also contribute to drug leakage and clearance. Therefore, research in this field should continue to optimize membrane separation and coating technology. It is worth mentioning that, compared with some of the harsher conditions faced by neutrophils, neutrophil membrane coating technology seems to be able to overcome some obstacles, which avoids the strict preparation conditions and sterilization methods from the extraction of living cells in the body and the transfusion back, as well as the high expense.

The characteristics of exosomes are between living cells and cell membranes, containing part of the genetic materials of the cell, including DNA, RNA, and protein, with "life" to a certain extent, to exert part of the function of neutrophils. It is like neutrophil-membrane-coated nanoparticles in size and structure, with great potential in the drug delivery field. Due to the different components of exosomes from source cells, their potential biological functions are also greatly different. Exosomes secreted by neutrophils have good chemotaxis of inflammation, which can cross the blood-brain barrier for the treatment of brain diseases, while exosomes secreted by dendritic cells have good immune recognition. Therefore, the selection of source cells is the premise for achieving the best therapeutic effect. However, it should be underlined that proteins and RNA that promote tumor metastasis can also utilize exosomes as escape vectors to avoid immune recognition of the body. Besides, large-scale production of exosomes is to be resolved for the limited extraction content. Although it has been reported that rapid purification can enable the large-scale production of therapeutic exosomes, further evaluation is needed to determine whether this technique applies to different cell types. Ultimately, the successful application of exosome-inspired drug delivery systems in clinics is still in progress. However, it is believed that shortly, such nanodrug delivery systems will benefit a vast number of patients.

Neutrophil-derived drug delivery systems show crucial importance in the nanotheranostics of tumors and inflammation. The advantages lie in prolonged circulation, augmented targeting, and better biostability. In this review, we provide a full description of neutrophil-derived nanodrug delivery in the management of tumors and inflammation. Although there are still many difficulties in clinical application, this paves the way for nanotheranostics of disease.

\section{Acknowledgments}

This work was supported by grants from Clinical research project of health industry of Shanghai Health Committee (201940200) and sponsored by Shanghai Sailing Program (21YF1435800).

\section{Disclosure}

The authors declare no conflict of interest. 


\section{References}

1. Neurath MF. Targeting immune cell circuits and trafficking in inflammatory bowel disease. Nat Immunol. 2019;20(8):970-979. doi:10.10 38/s41590-019-0415-0

2. Eming SA, Wynn TA, Martin P. Inflammation and metabolism in tissue repair and regeneration. Science. 2017;356(6342):1026-1030. doi:10.1126/science.aam7928

3. Kolaczkowska E, Kubes P. Neutrophil recruitment and function in health and inflammation. Nat Rev Immunol. 2013;13(3):159-175. doi: $10.1038 /$ nri3399

4. Soehnlein O, Libby P. Targeting inflammation in atherosclerosis from experimental insights to the clinic. Nat Rev Drug Discov. 2021;20:589-610.

5. Jaillon S, Ponzetta A, Di Mitri D, Santoni A, Bonecchi R, Mantovani A. Neutrophil diversity and plasticity in tumour progression and therapy. Nat Rev Cancer. 2020;20(9):485-503. doi:10.1038/ s41568-020-0281-y

6. Qin J, Chen DW, Hu HY, Cui Q, Qiao MX, Chen BY. Surface modification of RGD-Liposomes for selective drug delivery to monocytes/neutrophils in brain. Chem Pharm Bull. 2007;55(8):1192-1197. doi:10.1248/cpb.55.1192

7. Gu XY, Gao YZ, Wang P, et al. Nano-delivery systems focused on tumor microenvironment regulation and biomimetic strategies for treatment of breast cancer metastasis. $J$ Control Release. 2021;333:374-390. doi:10.1016/j.jconrel.2021.03.039

8. Liu YH, Zhou JY, Li Q, et al. Tumor microenvironment remodeling-based penetration strategies to amplify nanodrug accessibility to tumor parenchyma q. Adv Drug Deliver Rev. 2021;172:80-103. doi:10.1016/j.addr.2021.02.019

9. Din FU, Aman W, Ullah I, et al. Effective use of nanocarriers as drug delivery systems for the treatment of selected tumors. Int J Nanomed. 2017;12:7291-7309. doi:10.2147/IJN.S146315

10. van der Meel R, Sulheim E, Shi Y, Kiessling F, Mulder WJM, Lammers T. Smart cancer nanomedicine. Nat Nanotechnol. 2019;14 (11):1007-1017. doi:10.1038/s41565-019-0567-y

11. Choi KY, Han HS, Lee ES, et al. Hyaluronic acid-based activatable nanomaterials for stimuli-responsive imaging and therapeutics: beyond CD44-mediated drug delivery. Adv Mater. 2019;31 (34):1803549. doi:10.1002/adma.201803549

12. Stuart MAC, Huck WTS, Genzer J, et al. Emerging applications of stimuli-responsive polymer materials. Nat Mater. 2010;9(2):101-113. doi:10.1038/nmat2614

13. Sun QH, Zhou ZX, Qiu NS, Shen YQ. Rational design of cancer nanomedicine: nanoproperty integration and synchronization. $A d v$ Mater. 2017;29(14):1606628. doi:10.1002/adma.201606628

14. Stirland DL, Nichols JW, Miura S, Bae YH. Mind the gap: a survey of how cancer drug carriers are susceptible to the gap between research and practice. J Control Release. 2013;172(3):1045-1064. doi:10.1016/j.jconrel.2013.09.026

15. Gewin V, Giordani S. Designing nano-sized chemotherapy. Nature. 2021;593(7859):470. doi:10.1038/d41586-021-01322-2

16. Bourzac K. News feature: cancer nanomedicine, reengineered. Proc Natl Acad Sci USA. 2016;113(45):12600-12603. doi:10.1073/pnas.16 16895113

17. Peng CQ, Huang YY, Zheng J. Renal clearable nanocarriers: overcoming the physiological barriers for precise drug delivery and clearance. J Control Release. 2020;322:64-80. doi:10.1016/j.jconrel. 2020.03.020

18. He JY, Li CC, Ding L, et al. Tumor targeting strategies of smart fluorescent nanoparticles and their applications in cancer diagnosis and treatment. Adv Mater. 2019;31(40):1902409. doi:10.1002/ adma.201902409

19. Blanco E, Shen H, Ferrari M. Principles of nanoparticle design for overcoming biological barriers to drug delivery. Nat Biotechnol. 2015;33(9):941-951. doi:10.1038/nbt.3330
20. Mo R, Jiang TY, DiSanto R, Tai WY, Gu Z. ATP-triggered anticancer drug delivery. Nat Commun. 2014;5(1):3364. doi:10.1038/ncomms4364

21. Yuan P, Dou G, Liu T, et al. On-demand manipulation of tumorigenic microenvironments by nano-modulator for synergistic tumor therapy. Biomaterials. 2021;275:120956. doi:10.1016/j.biomaterials.2021.120956

22. Ma Z, Pi JX, Zhang Y, et al. Enhanced anticancer efficacy of dual drug-loaded self-assembled nanostructured lipid carriers mediated by pH-responsive folic acid and human-derived cell penetrating peptide dNP2. Pharmaceutics. 2021;13(5):600. doi:10.3390/pharmaceutics 13050600

23. Yu LX, Tang P, Nie CX, Hou Y, Haag R. Well-defined nanostructured biointerfaces: strengthened cellular interaction for circulating tumor cells isolation. Adv Healthc Mater. 2021;10(11):2002202. doi:10.10 02/adhm.202002202

24. Song J, Ju Y, Amarasena TH, et al. Influence of poly(ethylene glycol) molecular architecture on particle assembly and ex vivo particle-immune cell interactions in human blood. ACS Nano. 2021;15(6):10025-10038. doi:10.1021/acsnano.1c01642

25. Gregory AD, Houghton AM. Tumor-associated neutrophils: new targets for cancer therapy. Cancer Res. 2011;71(7):2411-2416. doi:10.1158/0008-5472.CAN-10-2583

26. Bi YH, Duan WX, Chen J, et al. Neutrophil decoys with anti-inflammatory and anti-oxidative properties reduce secondary spinal cord injury and improve neurological functional recovery. Adv Funct Mater. 2021;31(34):2102912. doi:10.1002/adfm.202102912

27. Li J, Long Y, Guo R, et al. Shield and sword nano-soldiers ameliorate rheumatoid arthritis by multi-stage manipulation of neutrophils. J Control Release. 2021;335:38-48. doi:10.1016/j. jconrel.2021.05.008

28. Han Y, Zhao RB, Xu F. Neutrophil-based delivery systems for nanotherapeutics. Small. 2018;14(42):e1801674. doi:10.1002/smll.20 1801674

29. Gustafson HH, Holt-Casper D, Grainger DW, Ghandehari H. Nanoparticle uptake: the phagocyte problem. Nano Today. 2015;10 (4):487-510. doi:10.1016/j.nantod.2015.06.006

30. Wu H, Wang MD, Liang L, et al. Nanotechnology for hepatocellular carcinoma: from surveillance, diagnosis to management. Small. 2021;17(6):e2005236. doi:10.1002/smll.202005236

31. Zhang WZ, Wang MZ, Tang W, et al. Nanoparticle-laden macrophages for tumor-tropic drug delivery. Adv Mater. 2018;30(50): 1805557. doi:10.1002/adma.201805557

32. Ahamad N, Kar A, Mehta S, et al. Immunomodulatory nanosystems for treating inflammatory diseases. Biomaterials. 2021;274:120875. doi:10.1016/j.biomaterials.2021.120875

33. Flavell RA, Sanjabi S, Wrzesinski SH, Licona-Limon P. The polarization of immune cells in the tumour environment by TGF beta. Nat Rev Immunol. 2010;10(8):554-567. doi:10.1038/nri2808

34. Ng LG, Ostuni R, Hidalgo A. Heterogeneity of neutrophils. Nat Rev Immunol. 2019;19(4):255-265. doi:10.1038/s41577-019-0141-8

35. Fang RH, Jiang Y, Fang JC, Zhang LF. Cell membrane-derived nanomaterials for biomedical applications. Biomaterials. 2017;12 8:69-83.

36. Dehaini D, Wei XL, Fang RH, et al. Erythrocyte-platelet hybrid membrane coating for enhanced nanoparticle functionalization. $A d v$ Mater. 2017;29(16):1606209. doi:10.1002/adma.201606209

37. Liu WC, Yan QW, Xia C, et al. Recent advances in cell membrane coated metal-organic frameworks (MOFs) for tumor therapy. J Mater Chem B. 2021;9(22):4459-4474. doi:10.1039/D1TB00453K

38. Hu CMJ, Zhang L, Aryal S, Cheung C, Fang RH, Zhang LF. Erythrocyte membrane-camouflaged polymeric nanoparticles as a biomimetic delivery platform. Proc Natl Acad Sci USA. 2011;108 (27):10980-10985. doi:10.1073/pnas.1106634108

39. Li LY, Zuo XX, Xiao YZ, Liu D, Luo H, Zhu HL. Neutrophil-derived exosome from systemic sclerosis inhibits the proliferation and migration of endothelial cells. Biochem Biophys Res Commun. 2020;526 (2):334-340. doi:10.1016/j.bbrc.2020.03.088 
40. Nauseef WM, Borregaard N. Neutrophils at work. Nat Immunol. 2014;15(7):602-611. doi:10.1038/ni.2921

41. Xue YA, Wu Y, Wang QQ, Xue LJ, Su ZG, Zhang C. Cellular vehicles based on neutrophils enable targeting of atherosclerosis. Mol Pharmaceut. 2019;16(7):3109-3120. doi:10.1021/acs.molpharmaceut. $9 \mathrm{~b} 00342$

42. Batrakova EV, Gendelman HE, Kabanov AV. Cell-mediated drug delivery. Expert Opin Drug Deliv. 2011;8(4):415-433. doi:10.1517/ 17425247.2011.559457

43. Pierige F, Serafini S, Rossi L, Magnani A. Cell-based drug delivery. Adv Drug Deliver Rev. 2008;60(2):286-295. doi:10.1016/j.addr.20 07.08.029

44. Xue JW, Zhao ZK, Zhang L, et al. Neutrophil-mediated anticancer drug delivery for suppression of postoperative malignant glioma recurrence. Nat Nanotechnol. 2017;12(7):692-700. doi:10.1038/ nnano.2017.54

45. Wang HJ, Liu Y, He RQ, et al. Cell membrane biomimetic nanoparticles for inflammation and cancer targeting in drug delivery. Biomater Sci. 2020;8(2):552-568. doi:10.1039/C9BM01392J

46. Wang M, Xin YF, Cao H, et al. Recent advances in mesenchymal stem cell membrane-coated nanoparticles for enhanced drug delivery. Biomater Sci. 2021;9(4):1088-1103. doi:10.1039/D0BM01164A

47. Wang KY, Lei YT, Xia DL, et al. Neutrophil membranes coated, antibiotic agent loaded nanoparticles targeting to the lung inflammation. Colloids Surf B Biointerfaces. 2020;188:110755. doi:10.1016/j.colsurfb.2019.110755

48. Zhao Q, Jiang D, Sun X, et al. Biomimetic nanotherapy: core-shell structured nanocomplexes based on the neutrophil membrane for targeted therapy of lymphoma. J Nanobiotechnol. 2021;19(1):179. doi:10.1186/s12951-021-00922-4

49. Zhang QZ, Dehaini D, Zhang Y, et al. Neutrophil membrane-coated nanoparticles inhibit synovial inflammation and alleviate joint damage in inflammatory arthritis. Nat Nanotechnol. 2018;13 (12):1182-1190. doi:10.1038/s41565-018-0254-4

50. Kang T, Zhu QQ, Wei D, et al. Nanoparticles coated with neutrophil membranes can effectively treat cancer metastasis. ACS Nano. 2017;11(2):1397-1411. doi:10.1021/acsnano.6b06477

51. Cully M. Exosome-based candidates move into the clinic. Nat Rev Drug Discov. 2021;20(1):6-7. doi:10.1038/d41573-020-00220-y

52. Agrawal M, Saraf S, Saraf S, et al. Recent strategies and advances in the fabrication of nano lipid carriers and their application towards brain targeting. J Control Release. 2020;321:372-415.

53. Zheng MB, Yue CX, Ma YF, et al. Single-step assembly of DOX/ ICG loaded lipid-polymer nanoparticles for highly effective chemo-photothermal combination therapy. ACS Nano. 2013;7 (3):2056-2067. doi:10.1021/nn400334y

54. Zhang YM, Liu YH, Liu Y. Cyclodextrin-based multistimuli-responsive supramolecular assemblies and their biological functions. Adv Mater. 2020;32(3):e1806158. doi:10.1002/adma. 201806158

55. Li YT, Tang JL, He LC, et al. Core-shell upconversion nanoparticle@metal-organic framework nanoprobes for luminescent/ magnetic dual-mode targeted imaging. Adv Mater. 2015;27 (27):4075-4080. doi:10.1002/adma.201501779

56. Zhang X, Machuki JO, Pan WZ, et al. Carbon nitride hollow theranostic nanoregulators executing laser-activatable water splitting for enhanced ultrasound/fluorescence imaging and cooperative phototherapy. ACS Nano. 2020;14(4):4045-4060. doi:10.1021/ acsnano.9b08737

57. Yang M, Xu ZX, Yan HL, et al. PD-L1 cellular nanovesicles carrying rapamycin inhibit alloimmune responses in transplantation. Biomater Sci. 2021;9(4):1246-1255. doi:10.1039/D0BM01798A

58. Chen FF, Wang GK, Griffin JI, et al. Complement proteins bind to nanoparticle protein Corona and undergo dynamic exchange in vivo. Nat Nanotechnol. 2017;12(4):387-393. doi:10.1038/nnano.2016.269
59. Zou YJ, Ito S, Yoshino F, Suzuki Y, Zhao L, Komatsu N. Polyglycerol grafting shields nanoparticles from protein corona formation to avoid macrophage uptake. ACS Nano. 2020;14 (6):7216-7226.

60. Cabral H, Matsumoto Y, Mizuno K, et al. Accumulation of sub-100 $\mathrm{nm}$ polymeric micelles in poorly permeable tumours depends on size. Nat Nanotechnol. 2011;6(12):815-823. doi:10.1038/nnano.2011.166

61. Yang GB, Phua SZF, Lim WQ, et al. A hypoxia-responsive albumin-based nanosystem for deep tumor penetration and excellent therapeutic efficacy. Adv Mater. 2019;31(25):1901513. doi:10.1002/adma.201901513

62. Gao A, Chen BF, Gao J, et al. Sheddable prodrug vesicles combating adaptive immune resistance for improved photodynamic immunotherapy of cancer. Nano Lett. 2020;20(1):353-362. doi:10.1021/ acs.nanolett.9b04012

63. Hayat SMG, Bianconi V, Pirro M, Jaafari MR, Hatamipour M, Sahebkar A. CD47: role in the immune system and application to cancer therapy. Cell Oncol. 2020;43(1):19-30. doi:10.1007/s13402-019-00469-5

64. Oroojalian F, Beygi M, Baradaran B, Mokhtarzadeh A, Shahbazi MA. Immune cell membrane-coated biomimetic nanoparticles for targeted cancer therapy. Small. 2021;17(12):e2006484. doi:10.1002/smll.202006484

65. Hu Q, Zhang XL, Jia LL, et al. Engineering biomimetic graphene nanodecoys camouflaged with the EGFR/HEK293 cell membrane for targeted capture of drug leads. Biomater Sci. 2020;8(20):5690-5697. doi:10.1039/D0BM00841A

66. Billingsley MM, Singh N, Ravikumar P, Zhang R, June $\mathrm{CH}$, Mitchell MJ. Ionizable lipid nanoparticle-mediated mRNA delivery for human CAR T cell engineering. Nano Lett. 2020;20(3): 1578-1589. doi:10.1021/acs.nanolett.9b04246

67. Chen Q, Wang C, Zhang X, et al. In situ sprayed bioresponsive immunotherapeutic gel for post-surgical cancer treatment. Nat Nanotechnol. 2019;14(1):89-97. doi:10.1038/s41565-018-0319-4

68. Nemeth T, Sperandio M, Mocsai A. Neutrophils as emerging therapeutic targets. Nat Rev Drug Discov. 2020;19(4):253-275.

69. Zhen X, Cheng PH, Pu KY. Recent advances in cell membrane-camouflaged nanoparticles for cancer phototherapy. Small. 2019;15(1):e1804105. doi:10.1002/smll.201804105

70. Zhang L, Zhang Y, Xue YN, et al. Transforming weakness into strength: photothermal-therapy-induced inflammation enhanced cytopharmaceutical chemotherapy as a combination anticancer treatment. Adv Mater. 2019;31:1805936.

71. Dong HQ, Li Y, Liu YQ, et al. A nano-immunotraining strategy to enhance the tumor targeting of neutrophils via in vivo pathogen-mimicking stimulation. Biomater Sci. 2019;7(12):52 38-5246. doi:10.1039/C9BM01278H

72. Ye B, Zhao B, Wang K, et al. Neutrophils mediated multistage nanoparticle delivery for prompting tumor photothermal therapy. J Nanobiotechnol. 2020;18(1):138. doi:10.1186/s12951-020-00682-7

73. Li M, Li SY, Zhou H, et al. Chemotaxis-driven delivery of nano-pathogenoids for complete eradication of tumors post-phototherapy. Nat Commun. 2020;11(1):1126. doi:10.1038/s41467020-14963-0

74. Chu DF, Zhao Q, Yu J, Zhang FY, Zhang H, Wang ZJ. Nanoparticle targeting of neutrophils for improved cancer immunotherapy. $A d v$ Healthc Mater. 2016;5(9):1088-1093. doi:10.1002/adhm.201500998

75. Luo X, Liu MQ, Hu L, et al. Targeted delivery of pixantrone to neutrophils by poly(sialic acid)-p-octadecylamine conjugate modified liposomes with improved antitumor activity. Int $J$ Pharmaceut. 2018;547(1-2):315-329.

76. Li S, Li M, Huo S, et al. Voluntary-opsonization-enabled precision nanomedicines for inflammation treatment. Adv Mater. 2020;33(3): e2006160. doi:10.1002/adma.202006160

77. Su YJ, Wang TT, $\mathrm{Su}$ YN, et al. A neutrophil membrane-functionalized black phosphorus riding inflammatory signal for positive feedback and multimode cancer therapy. Mater Horiz. 2020;7(2):574-585. doi:10.1039/C9MH01068H 
78. Cao X, Hu Y, Luo S, et al. Neutrophil-mimicking therapeutic nanoparticles for targeted chemotherapy of pancreatic carcinoma. Acta Pharmacol Sin B. 2019;9(3):575-589. doi:10.1016/j.apsb.2018.12.009

79. Wang JS, Gu XM, Ouyang YQ, et al. Engineering of neutrophil membrane camouflaging nanoparticles realizes targeted drug delivery for amplified antitumor therapy. Int J Nanomed. 2021;16:1175-1187. doi:10.2147/IJN.S288636

80. Zhang C, Zhang L, Wu W, et al. Artificial super neutrophils for inflammation targeting and $\mathrm{HClO}$ generation against tumors and infections. $A d v$ Mater. 2019;31(19):e1901179. doi:10.1002/adma.201901179

81. Wang J, Tang W, Yang M, et al. Inflammatory tumor microenvironment responsive neutrophil exosomes-based drug delivery system for targeted glioma therapy. Biomaterials. 2021;273:120784. doi:10.10 16/j.biomaterials.2021.120784

82. Zhang P, Zhao Q, Shi MS, et al. Fe3O4@TiO2-laden neutrophils activate innate immunity via photosensitive reactive oxygen species release. Nano Lett. 2020;20(1):261-271. doi:10.1021/acs.nanolett.9b03777

83. Zhang CY, Dong XY, Gao J, Lin WJ, Liu Z, Wang ZJ. Nanoparticleinduced neutrophil apoptosis increases survival in sepsis and alleviates neurological damage in stroke. Sci Adv. 2019;5(11):eaax7964. doi:10.1126/sciadv.aax7964

84. Tang CM, Wang C, Zhang Y, et al. Recognition, intervention, and monitoring of neutrophils in acute ischemic stroke. Nano Lett. 2019;19(7):4470-4477. doi:10.1021/acs.nanolett.9b01282

85. Zhou X, Cao X, Tu H, Zhang ZR, Deng L. Inflammation-targeted delivery of celastrol via neutrophil membrane-coated nanoparticles in the management of acute pancreatitis. Mol Pharmaceut. 2019;16 (3):1397-1405. doi:10.1021/acs.molpharmaceut.8b01342
86. Zhang L, Cheng Q, Li C, Zeng X, Zhang XZ. Near infrared light-triggered metal ion and photodynamic therapy based on AgNPs/porphyrinic MOFs for tumors and pathogens elimination. Biomaterials. 2020;248:120029. doi:10.1016/j.biomaterials.2020.120029

87. Tang C, Wang Q, Li K, et al. A neutrophil-mimetic magnetic nanoprobe for molecular magnetic resonance imaging of stroke-induced neuroinflammation. Biomater Sci. 2021;9(15):5247-5258. doi:10.10 39/D1BM00566A

88. Gao J, Wang S, Dong X, Leanse LG, Dai T, Wang Z. Co-delivery of resolvin D1 and antibiotics with nanovesicles to lungs resolves inflammation and clears bacteria in mice. Commun Biol. 2020;3 (1):680. doi:10.1038/s42003-020-01410-5

89. Gao J, Dong XY, Su YJ, Wang ZJ. Human neutrophil membrane-derived nanovesicles as a drug delivery platform for improved therapy of infectious diseases. Acta Biomater. 2021;123:354-363. doi:10.1016/j.actbio.2021.01.020

90. Dong XY, Gao J, Zhang CY, Hayworth C, Frank M, Wang ZJ. Neutrophil membrane-derived nanovesicles alleviate inflammation to protect mouse brain injury from ischemic stroke. ACS Nano. 2019;13(2):1272-1283.
International Journal of Nanomedicine

\section{Publish your work in this journal}

The International Journal of Nanomedicine is an international, peerreviewed journal focusing on the application of nanotechnology in diagnostics, therapeutics, and drug delivery systems throughout the biomedical field. This journal is indexed on PubMed Central, MedLine, CAS, SciSearch ${ }^{\circledR}$, Current Contents ${ }^{\circledR} /$ Clinical Medicine,

\section{Dovepress}

Journal Citation Reports/Science Edition, EMBase, Scopus and the Elsevier Bibliographic databases. The manuscript management system is completely online and includes a very quick and fair peer-review system, which is all easy to use. Visit http://www.dovepress.com/ testimonials.php to read real quotes from published authors. 